\title{
The asymmetric eddy-background flow interaction in the North Pacific storm track
}

Article

Accepted Version

Zhao, Y.-B., Liang, X. S., Guan, Z. and Hodges, K. I. (2019)

The asymmetric eddy-background flow interaction in the North

Pacific storm track. Quarterly Journal of the Royal

Meteorological Society, 145 (719). pp. 575-596. ISSN 1477-

870X doi: https://doi.org/10.1002/qj.3453 Available at

https://centaur.reading.ac.uk/80948/

It is advisable to refer to the publisher's version if you intend to cite from the work. See Guidance on citing.

To link to this article DOI: http://dx.doi.org/10.1002/qj.3453

Publisher: Royal Meteorological Society

All outputs in CentAUR are protected by Intellectual Property Rights law, including copyright law. Copyright and IPR is retained by the creators or other copyright holders. Terms and conditions for use of this material are defined in the End User Agreement.

\section{www.reading.ac.uk/centaur}

\section{CentAUR}

Central Archive at the University of Reading 
Reading's research outputs online 


\section{Quarterly Journal of the}

The asymmetric eddy-background flow interaction in the North Pacific storm track

\begin{tabular}{|c|c|}
\hline Journal: & QJRMS \\
\hline Manuscript ID & QJ-18-0042.R2 \\
\hline Wiley - Manuscript type: & Research Article \\
\hline $\begin{array}{r}\text { Date Submitted by the } \\
\text { Author: }\end{array}$ & $\mathrm{n} / \mathrm{a}$ \\
\hline Complete List of Authors: & $\begin{array}{l}\text { Zhao, Yuan-Bing; Nanjing University of Information Science and } \\
\text { Technology, } \\
\text { Liang, X. San; Nanjing University of Information Science and Technology } \\
\text { Guan, Zhaoyong; Nanjing University of Information Science and } \\
\text { Technology } \\
\text { Hodges, Kevin; The University of Reading, Dept. of Meterology }\end{array}$ \\
\hline Keywords: & $\begin{array}{l}\text { eddy-mean flow interaction, extra-tropical cyclone, feature tracking, } \\
\text { multiscale energetics, canonical energy transfer }\end{array}$ \\
\hline Country Keywords: & China \\
\hline
\end{tabular}


1 The asymmetric eddy-background flow interaction in the North Pacific storm track

2 Yuan-Bing Zhao ${ }^{1}$, X. San Liang ${ }^{12 *}$, Zhaoyong Guan ${ }^{1}$, and Kevin I. Hodges ${ }^{3}$

3

$4 \quad{ }^{1}$ School of Atmospheric Sciences, Nanjing University of Information Science and

5 Technology, Nanjing, Jiangsu, 210044, China

$6{ }^{2}$ School of Marine Sciences, Nanjing University of Information Science and Technology,

7 Nanjing, Jiangsu, 210044, China

$8{ }^{3}$ Department of Meteorology, National Centre for Earth Observation, University of

$9 \quad$ Reading, Reading, UK

10

11 Correspondence

12 X. San Liang, Nanjing Center for Ocean-Atmosphere Dynamical Studies, Nanjing,

13 Jiangsu, 210044, China.

14 E-mail: x.san.liang@gmail.com

15

16 Running head

17 Asymmetric eddy-mean flow interaction

18

19 


\section{Abstract}

21 Using a recently developed methodology, namely, the multiscale window transform

22 (MWT), and the MWT-based theory of canonical transfer and localized multiscale 23 energetics analysis, we investigate in an eddy-following way the nonlinear 24 eddy-background flow interaction in the North Pacific storm track, based on the ERA40 25 reanalysis data from ECWMF. It is found that more than $50 \%$ of the storms occur on the 26 northern flank of the jet stream, about $40 \%$ are around the jet center, and very few (less 27 than 5\%) happen on the southern flank. For storms near or to the north of the jet center, 28 their interaction with the background flow is asymmetric in latitude. In higher latitudes, 29 strong downscale canonical available potential energy transfer happens, especially in the 30 middle troposphere, which reduces the background baroclinicity and decelerates the jet; 31 in lower latitudes, upscale canonical kinetic energy transfer intensifies at the jet center, 32 accelerating the jet and enhancing the middle-level baroclinicity. The resultant effect is 33 that the jet strengthens but narrows, leading to an anomalous dipolar pattern in the fields 34 of background wind and baroclinicity. For the storms on the southern side of the jet, the 35 baroclinic canonical transfer is rather weak. On average, the local interaction begins from 36 about 3 days before a storm arrives at the site of observation, achieves its maximum as 37 the storm arrives, and then weakens. 
47 been shown that the atmospheric storm track generally coincides with the tropospheric

48 jet, where the baroclinicity is the strongest (e.g., Chang et al., 2002; Lee and Kim, 2003).

49 Within the storm track, synoptic eddies are often generated in the jet and then interact 50 with it.

51

52 eddies and the jet stream. In most early studies, the focus was on the theoretical aspects

53 based on idealized models, e.g., those with small-amplitude perturbations in the form of

54 sinusoidal waves and a zonally homogeneous, stationary background flow (in many cases

55 also meridionally independent) (Andrews and McIntyre, 1978; Charney and Drazin, 56 1961; Dickinson, 1969; Eliassen and Palm, 1961; Lindzen and Holton, 1968; Plumb, 57 1985). However, in the real atmosphere the background flow is by nature temporally 58 varying and zonally asymmetric; especially in midlatitudes, due to the large scale 59 topography and land-sea contrasts (e.g., Lee and Kim, 2003; Li and Wettstein, 2011), 60 whilst the eddies are rather localized in space and time with finite amplitudes (Blackmon 
61 et al., 1984; Catto et al., 2010; Chang, 1993; Lim and Wallace, 1991; Simmons and

62 Hoskins, 1978). In more recent studies, with the advent of observational and reanalysis

63 data, discrepancies have been found between the previously proposed theories and the

64 observations. For example, based on the previous theories it is supposed that baroclinic

65 energy transfer associated with baroclinic instability is bottom-trapped (Edmon et al.,

66 1980; Green, 1960; Hoskins et al., 1985; Lindzen and Farrell, 1980; Pierrehumbert and

67 Swanson, 1995), whereas observational energetics diagnostics have implied that

68 midlatitude synoptic systems have their maximum baroclinic energy transfer in middle

69 levels (e.g., Chang et al., 2002). The spatial structure of the interaction is still a problem

70 yet to be explored.

71 During the past two decades, eddy-mean flow interaction has been extensively

72 discussed, especially in studies of annular modes (Barnes and Hartmann, 2011; Burrows

73 et al., 2017; Chen and Plumb, 2009; Gerber and Vallis, 2007; Kidston et al., 2010;

74 Kidston and Vallis, 2012; Lorenz, 2014; Lorenz and Hartmann, 2001; Robinson, 2000;

75 Zhang et al., 2012, to name but a few). But, most of these studies have focused on the

76 zonal-mean and climatologically statistical aspects. Rarely considered is the interaction

77 between a typical individual eddy and the mean flow, except for a few studies such as

78 Gerber and Vallis (2007), who argued that if the eddy is generated in the jet, the vertical

79 wind shear is first reduced by the heat flux in the baroclinic development stage, and then

80 in the decaying stage the jet is strengthened by upgradient momentum transfer through

81 the wave meridional propagation (the net propagation of baroclinic wave activity away 
82 from the jet gives momentum fluxes into the jet). On the whole, the jet structure is

83 maintained in the presence of surface friction. Alternatively, if the eddy is growing on the

84 shoulders of the jet, the baroclinic development stage is similar to that of the eddy

85 growing in the jet. However, the meridional wave propagation is then limited aloft, and,

86 as a result, the shear is reduced locally rather than over the entire baroclinic zone. Similar

87 studies are also reported in Zhang et al. (2012) based on an idealized $\beta$-plane

88 quasi-geostrophic model. As remarked by Vallis and Gerber (2008), the applicability of

89 these results to the real atmosphere still needs verification.

90 We remark that, earlier on, a classical way to look at the eddy-mean flow interaction

91 is with the "barotropic generation rate (Mak and Cai 1989; Cai and Mak 1990; Rivière et

92 al. 2003) and "barocilinc generation rate" (Cai and Mak 1990; Rivière et al. 2004). The

93 merit of this method is that if the eddy structure, the deformation of the jet, and the

94 positional relationship between the eddy and the jet are known, then the energy exchange

95 between the jet and the eddy can be easily determined (especially with the barotropic

96 generation rate). But here this method is difficult to be applied, as it is derived in the

97 quasi-geostrophic (QG) framework with the small amplitude assumption; besides, it does

98 not tell how eddies would modify the mean flow.

99 In this study we apply a newly developed methodology, which is capable of 100 handling these nonlinear problems in a generic sense, to diagnose the two-way 101 interactions between individual midlatitude synoptic eddies and the background flow in 102 an eddy-following way, in the hope of unraveling what is really happening locally in the 
103 North Pacific storm track. The methodology includes a functional analysis apparatus, 104 namely, the Multiscale Window Transform (MWT, Liang and Anderson 2007), and the

105 MWT-based localized multiscale energy and vorticity analysis (MS-EVA, Liang 2016).

106 The rest of this paper is organized as follows. Section 2 describes the data used in

107 this study. In section 3, we briefly introduce the MWT, MS-EVA, and the Lagrangian

108 tracking and compositing method, and in section 4, we set up the MS-EVA application

109 with the data. In the following sections $(5,6,7$, and 8), the composite reconstructed fields

110 and a detailed eddy-mean flow interaction analysis are presented. The study is

111 summarized in section 9.

112 2. Data

113 We use for our study the 40-year European Centre for Medium-Range Weather

114 Forecasts (ECWMF) ReAnalysis (ERA40) dataset (Uppala et al., 2005), which is

115 constrained by observations using a three-dimensional variational (3D-Var) data

116 assimilation system. It has been used because of its length (45 years), spatial resolution

117 (approximately $1.1^{\circ} \times 1.1^{\circ}$ in the tropics (T159), 35 vertical levels below $100 \mathrm{hPa}$ ), and

118 temporal resolution $(6 \quad$ h). More information can be found at

119 http://apps.ecmwf.int/datasets/data/era40-daily/levtype=pl/. Here we need the velocity

120 components (u, v, and $\omega)$, geopotential $(\phi)$, and temperature $(T)$ for the whole period

121 from September 1957 to July 2002. 
122

123

124

125

126

127

128

129

130

131

132

134

135

136

137 three-window decomposition for testing purpose. For easy reference, they are denoted

138 and will be referred to as windows $\varpi=0,1, \ldots$ Given a time series $T(t)$ with $N$ steps,

139 application of MWT yields two types of quantities: one is the MWT transform

140 coefficients, $\hat{T}_{n}^{\sim \varpi}(n=1,2, \cdots, N$, corresponding to the time location in $t)$, another the

141 multiscale window reconstruction (MWR), $T^{\sim \varpi}(t) . \quad T^{\sim \varpi}(t)$ is just like the 
142 low/high-pass filtered quantity. For example, in the two-window decomposition in this 143 study, the series $T(t)$ is decomposed into $T=T^{\sim 0}(t)+T^{\sim 1}(t)$, where $T^{\sim 0}$ stands for

144 the background field, and $T^{\sim 1}$ the transient (or synoptic) eddy field. MWT and MWR 145 form a transform-reconstruction pair, but they are distinctly different concepts, with the

146 former defined in phase space while the latter in physical space (here $t$ ), just like the

147 Fourier transform and inverse Fourier transform. The MWR of $T(t)$ on the synoptic-scale

148 window, for example, corresponds to a high-pass filtered signal. The lack of transform

149 coefficient $\hat{T}_{n}^{\sim \varpi}$ in traditional filters makes it impossible to represent multiscale energy ${ }^{1}$.

150 The common practice that simply takes multiscale energy as the square of filtered

151 variables is a conceptual mistake which, unfortunately, has frequently appeared in the

152 literature. But here with $\hat{T}_{n}^{\sim \varpi}$ it has been established that multiscale energy can be

153 precisely represented as the product of the MWT coefficients (up to some constant

154 multiplier). For example, the transient eddy energy extracted from $T(t)$ is simply $\left(\hat{T}_{n}^{\sim 1}\right)^{2}$

155 multiplied by some constant.

156 With MWT, the available potential energy (APE) and kinetic energy (KE) densities

157 on window $\varpi$ at time location $n$, written as $A_{n}^{\varpi}$ and $K_{n}^{\varpi}$, can be obtained, and their

158 evolution equations derived by applying MWT to the primitive governing equations.

159 Details are referred to Appendix A; the following is a symbolic representation (location $n$

160 in the subscript omitted henceforth for simplicity):

\footnotetext{
${ }^{1}$ Note one cannot write it in terms of the filtered quantities such as $\left[T^{\sim 1}(t)\right]^{2}$, as multiscale energy is a concept in phase space. (Think about that in a Fourier spectrum.) Refer to Appendix A for more explanation.
} 


$$
\begin{aligned}
& \frac{\partial A^{\varpi}}{\partial t}+\nabla \cdot \mathbf{Q}_{A}^{\varpi}=\Gamma_{A}^{\varpi}-b^{\varpi}+\mathrm{S}_{A}^{\varpi}+\mathrm{F}_{A}^{\varpi} \\
& \frac{\partial K^{\varpi}}{\partial t}+\nabla \cdot \mathbf{Q}_{K}^{\varpi}=\Gamma_{K}^{\varpi}-\nabla \cdot \mathbf{Q}_{P}^{\varpi}+b^{\varpi}+\mathrm{F}_{K}^{\varpi}
\end{aligned}
$$

161 where $\varpi=0$ and 1 stand for the two scale windows in this study, i.e., the basic flow

162 window and the eddy window. The Q's with subscripts A, K, and P are, respectively,

163 fluxes of APE, KE, and pressure, the $\Gamma$ terms are the transfer of energy (APE or KE) to

164 the designated scale window $\varpi$ from other windows, $b$ is buoyancy conversion, and $\mathrm{F}$

165 denotes the contribution from dissipation/diffusion. Explicit expressions and detailed

166 physical interpretations are referred to Appendix A, Table A.1. Note that all the terms are

167 localized both in space and in time; in other words, they are all four-dimensional field

168 variables, distinguished notably from the classical formalisms in which localization is lost

169 in at least one dimension of space-time in order to achieve scale decomposition.

170 Processes localized in space and time are thus naturally embedded in (1) and (2).

171 Although the terms in (1) and (2) have the conventional names (e.g., Orlanski and

172 Katzfey 1991; Chang 1993), they are actually distinctly different from those in traditional

173 formalisms. The most distinct terms are $\Gamma_{A}^{\varpi}$ and $\Gamma_{K}^{\varpi}$, which are the processes that we are

174 most interested in for this study. In appendix A we will see that they both have a Lie

175 bracket form, and possess the property of Jacobian identity, reminiscent of the Poisson

176 bracket in Hamiltonian dynamics; they also satisfy a detailed balance relation. Most

177 importantly, they sum to zero over scale window $\varpi$ and location $n$. This conservation

178 property, though simple to state, does not hold in previous energetic formalisms. To 
179 distinguish it from those that may have been encountered in the literature, the above

180

181

182

183

184

185

186

187

188

189

190

191

192

193

194

195

196

197

198

199

transfer is termed canonical transfer.

The canonical transfers $\left(\Gamma_{A}^{\Phi}\right.$ and $\left.\Gamma_{K}^{\Phi}\right)$ in (1) and (2) are very important. Particularly, the mean-to-eddy parts of them (written as $\Gamma_{A}^{0 \rightarrow 1}$ and $\Gamma_{K}^{0 \rightarrow 1}$ ) correspond to the two important geophysical fluid flow processes, i.e., baroclinic instability and barotropic instability of the mean flow (see Liang and Robinson, 2007), though whether they are equivalent is still in dispute (e.g., Farrell, 1984; 1985; 1989; Plumb, 1983). For mnemonic reason, in the following, $\Gamma_{A}^{0 \rightarrow 1}$ and $\Gamma_{K}^{0 \rightarrow 1}$ may be referred to as, respectively, baroclinic and barotropic canonical transfers. Conversely, the eddy-to-mean parts, written as $\Gamma_{\mathrm{A}}^{1 \rightarrow 0}$ and $\Gamma_{\mathrm{K}}^{1 \rightarrow 0}$, can be used to investigate the eddy feedback processes. They respectively correspond to the baroclinic and barotropic feedback mechanisms. See Figure A1 for an illustration.

\subsection{Eddy tracking and compositing}

The methods used in this study also include an eddy tracking and compositing technique. Because of the migration of the eddies and the inhomogeneity of the background flow, an eddy-following approach is needed to investigate the localized interaction. Here the tracking algorithm developed by Hodges (1995) is used to fulfil this task. In practical use, the maxima of the $850-\mathrm{hPa}$ relative vorticity $\left(\xi_{850}\right)$ are chosen as the indicator of the storm center (or feature point), which forms the nodes of the storm trajectory (Hoskins and Hodges, 2002). Besides, before tracking the vorticity maxima associated with weather storms it is necessary to remove the background field, i.e., the 
200 slowly varying, large spatial scale part of the field (Anderson et al. 2003). Here, we use 201 the synoptic vorticity reconstructed by the MWT as the indicator. That is to say, the 202 eddies tracked are storms in the synoptic scale window. Following previous studies, the 203 cyclogenesis time is defined as the first time when $\xi_{850}$ exceeds $1 \times 10^{-5} \mathrm{~s}^{-1}$. In this 204 study, we only select the long-lived storms whose $\xi_{850}$ remains larger than $1 \times 10^{-5} \mathrm{~s}^{-1}$ 205 for at least 4 days after their genesis. Also, only storms with a maximum $\xi_{850}$ greater 206 than $5 \times 10^{-5} \mathrm{~s}^{-1}$ are retained for the analysis. Finally, trajectories of 2189 cold-month 207 (from October to April) storms in the North Pacific storm-track area $\left[20^{\circ} \mathrm{N}-70^{\circ} \mathrm{N}\right.$; $\left.208120^{\circ} \mathrm{E}-240^{\circ} \mathrm{E}\right]$ are obtained. A sample of the selected trajectories are displayed in Figure 2091.

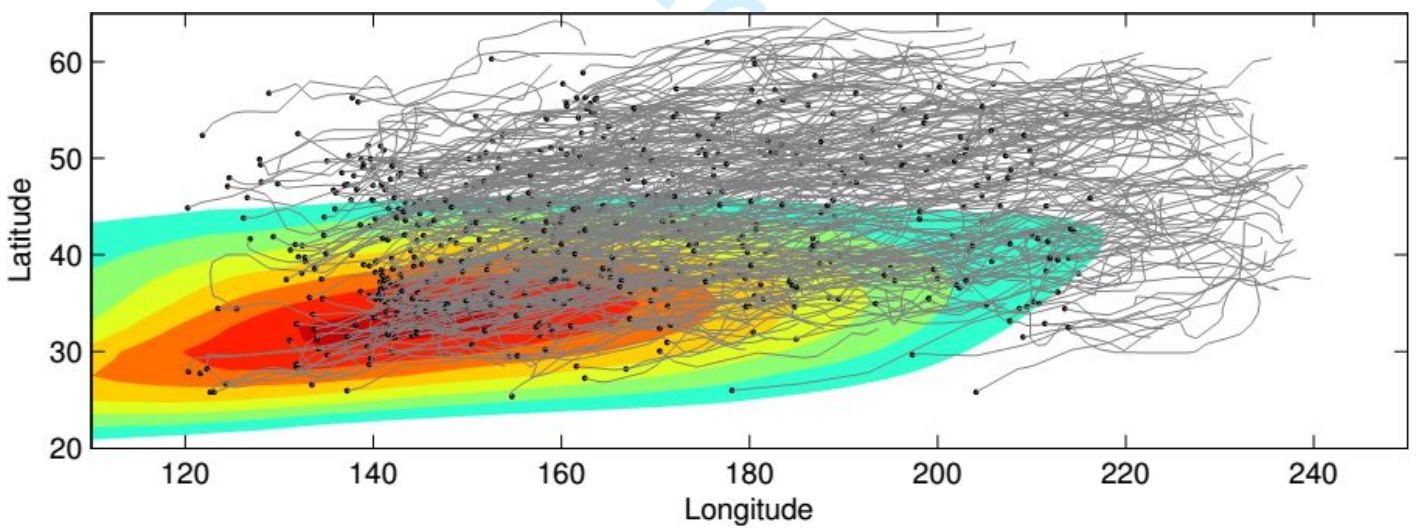

211 Figure 1. Sample of storm trajectories based on $\xi_{850}$. The gray lines stand for trajectories,

212 with black dots indicating their respective starting points, and the shaded area for the

213 climatological wintertime $300-\mathrm{hPa}$ zonal wind, starting from $20 \mathrm{~m} \mathrm{~s}^{-1}$ with an interval of $2145 \mathrm{~m} \mathrm{~s}^{-1}$.

215 The cyclone trajectories obtained are used for the compositing of the spatial 216 structures. Considering the large case-to-case variability (Sinclair and Revell, 2000), we 
217 use the compositing method with a large number of individual storms in order to get a 218 statistical result. A detailed description of the methodology can be found in Bengtsson et 219 al. (2007) for a radial sampling grid since modified for a rectangular sampling grid. In 220 brief, it consists of the following procedures:

$221 *$ First, select the tracks to be used. (In the present study, the storms of interest are 222 the 2189 long-lived strong storms.)

$223 *$ Second, create a rectangular grid centered on the equator with uniform grid 224 spacing and chosen side length (respectively 0.5 and 40 degrees here).

* Third, rotate the grid to the storm center and rotate it to align with the direction of 226 the storm propagation, which is determined by the displacement of the storm center using 227 a second-order, central differencing scheme. Using the rotated composite can reduce the 228 impact of the difference in the storm propagation direction on the composite storm 229 structure.

* Finally, the required field is sampled on to the rectangular grid, for each storm at 231 each time step along the full lifecycle of the storms and then averaged over the selected 232 storms at selected stages of the lifecycle. For the averaging, we need a reference time for 233 the lifecycle. In this study, the instant of maximal intensity is chosen as $t=0$, and then 234 the time is measured as offset to this reference time. For example, -4 (4) stands for 4 time 235 steps (or $24 \mathrm{~h}$ ) before (after) the storm reaches its maximal intensity. In this study, the 236 standard deviation is used to show the case-to-case variability within the composites. 


\section{4. MS-EVA setup}

238 The analysis begins with a determination of the scale window bounds. In this study, 239 we need two windows: a synoptic-scale window and a low-frequency background flow 240 window. According to previous studies, these windows are set to be bounded by a period 241 of 16 days (in MWT, the number of time steps is required to be a power of 2). We have 242 also tried 8 days as the window bound and found that the synoptic signal cannot be 243 completely separated from the total fields. This is essentially the same as Deng and Mak 244 (2006), who applied a 15-day high-pass filter in their diagnostics. Besides, Anderson et 245 al. (2003) observed that band-pass filtering with a time period of 2.5-6 days (e.g., 246 Blackmon, 1976) may have a detrimental impact on individual weather systems (see also 247 Chang, 1993), and a 20-day high-pass filter is a good choice. 

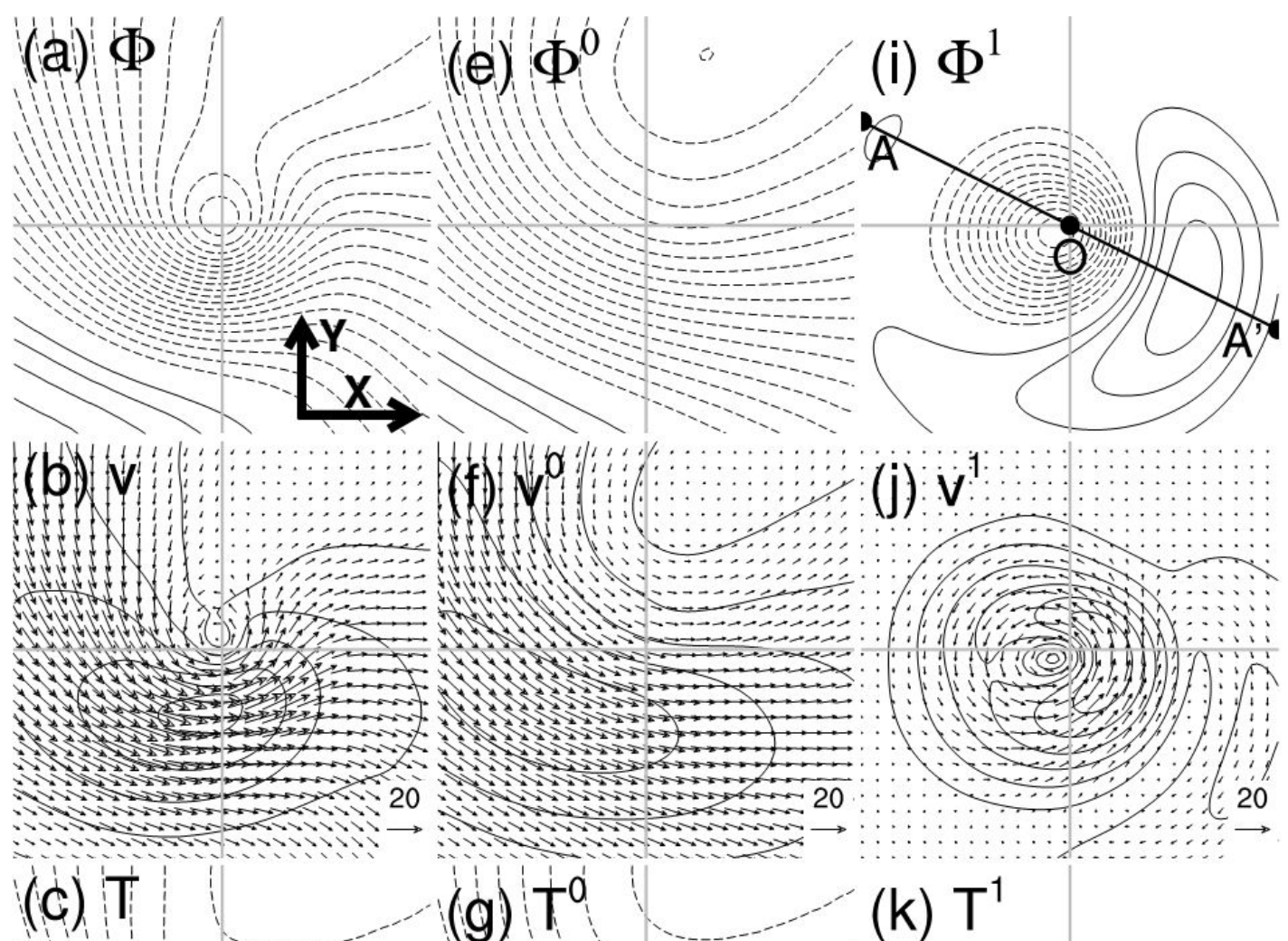

(g) $\mathrm{t}^{\mathrm{o}}$

(k) $\mathrm{T}^{1}$

249
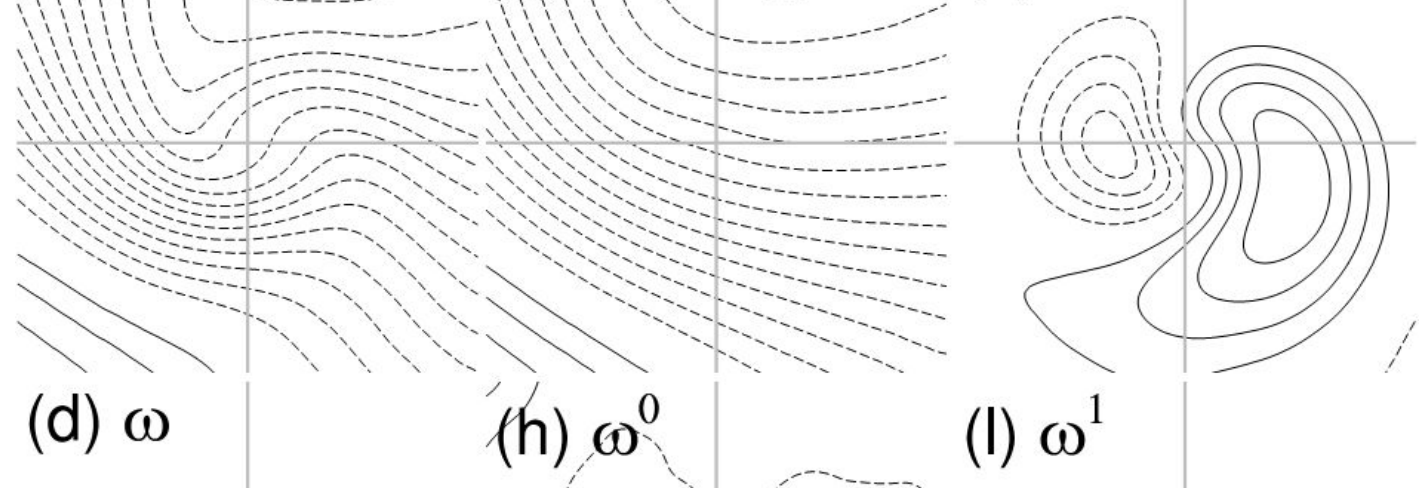

(d) $\omega$

(h) $\omega^{0}$

(I) $\omega^{1}$

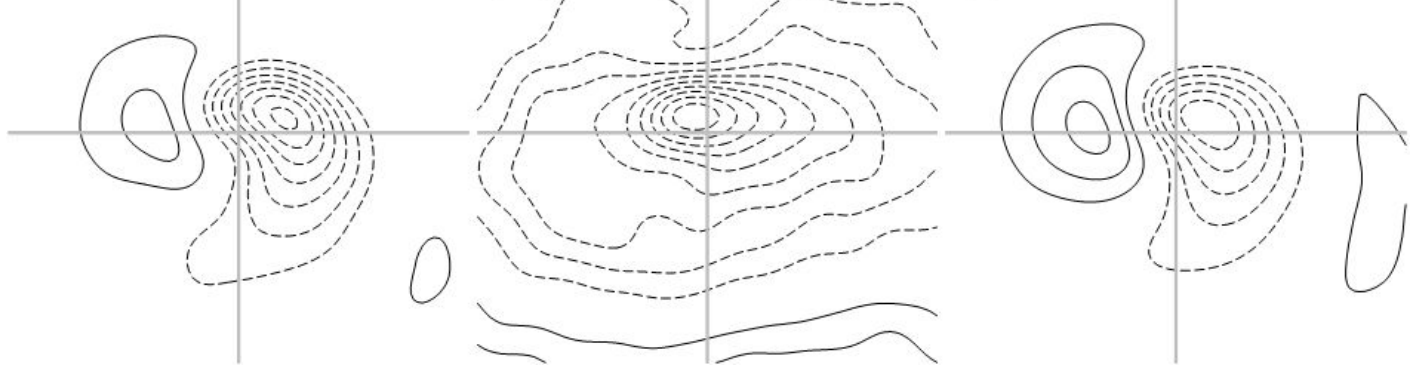

250 Figure 2. Left column: distributions of the 500-hPa composite original fields at the

251 maximal intensity time ( $\mathrm{t}=0$ ): (a) geopotential anomalies (contour interval $200 \mathrm{~m}^{2} \mathrm{~s}^{-2}$ ), (b)

252 wind vectors $\left(\mathrm{m} \mathrm{s}^{-1}\right.$ ) and speeds (contour, contour interval $5 \mathrm{~m} \mathrm{~s}^{-1}$ ), (c) temperature 
253 anomalies (contour interval $1 \mathrm{~K}$ ), and (d) vertical velocity (contour interval $0.04 \mathrm{~Pa} \mathrm{~s}^{-1}$ ).

254 Middle column: same as the left column, but for the background fields, with contour 255 intervals of (e) $200 \mathrm{~m}^{2} \mathrm{~s}^{-2}$, (f) $3 \mathrm{~m} \mathrm{~s}^{-1}$, (g) $1 \mathrm{~K}$, and (h) $0.004 \mathrm{~Pa} \mathrm{~s}^{-1}$. Right column: same 256 as the left column, but for the synoptic fields, with contour intervals of (i) $100 \mathrm{~m}^{2} \mathrm{~s}^{-2}$, (j) 2 $257 \mathrm{~m} \mathrm{~s}^{-1}$, (k) $0.5 \mathrm{~K}$, and (1) $0.04 \mathrm{~Pa} \mathrm{~s}^{-1}$. Negative contours are dashed and the zero contour is 258 omitted. The composite storm propagates eastward. Each subplot is in a $40^{\circ} \times 40^{\circ}$ 259 rectangular grid. The composites are based on the 2185 cold-month (October-April) 260 storms; same below. The corresponding distributions of the standard deviation are given 261 in Figure S3 in the supplementary file.

262 5. Composite structure of the original and reconstructed fields

263 In this section, we briefly describe the composite structures of the original and 264 reconstructed fields. Since the structure of each composite field changes little throughout 265 the life of the composite storm, only the maps at the storm's maximal intensity time $(\mathrm{t}=$ 266 0) are presented (note that the storm strength changes significantly throughout the life; 267 see Figures S1 and S2 in the supplementary file). Figure 2 shows the horizontal 268 distributions of the composite fields at $500 \mathrm{hPa}$. The side length of each subplot is 40 269 degrees at the equator (equivalent to $4447 \mathrm{~km}$ ). The composite system moves from the 270 left to the right. For convenience, the along-propagation direction will be referred to as 271 the $x$-direction, and the $y$-direction and $z$-direction are determined through the right-hand 272 rule, with $z$ directed upward. 
275 is a trough with closed isopleths (Figure 2a). Correspondingly, a cyclonic circulation 276 exists on the map of the horizontal wind $\left(\boldsymbol{v}_{\mathrm{h}}\right.$, Figure $\left.2 \mathrm{~b}\right)$. The distribution of the wind 277 speed is quite inhomogeneous around the storm center, with strong winds occurring in the

278 south part where the contours of geopotential anomalies are dense. The composite field of 279 temperature anomalies $(T)$ exhibits a wave-like pattern (Figure 2c), with the temperature 280 trough and ridge located to the west and east of the storm center, respectively. In terms of 281 the vertical velocity $(\omega)$, it shows a dipole pattern (Figure $2 \mathrm{~d})$, with upward $(\omega<0)$ and 282 downward $(\omega>0)$ motions located ahead and behind the geopotential trough, 283 respectively; the upward motion is much stronger than the downward motion. The 284 features of these composite fields generally agree with those in previous studies 285 (Bengtsson et al., 2009; Catto et al., 2010; Dacre et al., 2012; Field and Wood, 2007).

We first look at the original fields. On the map of geopotential anomalies $(\phi)$, there

\subsection{The multiscale window reconstructed fields}

We now look at the composites of the multiscale fields reconstructed by the MWT.

To our knowledge, few studies have explored the storm structure in this way. Figures $2 \mathrm{e}-2 \mathrm{~h}$ show the composite maps of the background fields. We see that the contours of geopotential anomalies $\left(\phi^{\sim 0}\right)$ are generally northwest-southeastward distributed (Figure 2e), and the closed center appearing in the original field (Figure 2a) has been removed. The background wind maximum (i.e., the jet stream) is located in the south (Figure 2f).

This is why the maximum wind occurs in the southern part of the storm on the original

\subsection{The original fields}


294 map (Figure $2 \mathrm{~b}$ ). For temperature anomaly $\left(T^{\sim 0}\right.$ ), its distribution (Figure $2 \mathrm{~g}$ ) is similar to 295 that of $\phi^{\sim 0}$. Regarding $\omega^{\sim 0}$, it is negative within almost the whole domain (Figure $2 \mathrm{~h}$ ), 296 implying that the storms are generated and evolve within an environment of upward 297 motion. The magnitude of $\omega^{\sim 0}$ is about $10 \%$ of the original field (note the difference in 298 the contour interval between them).
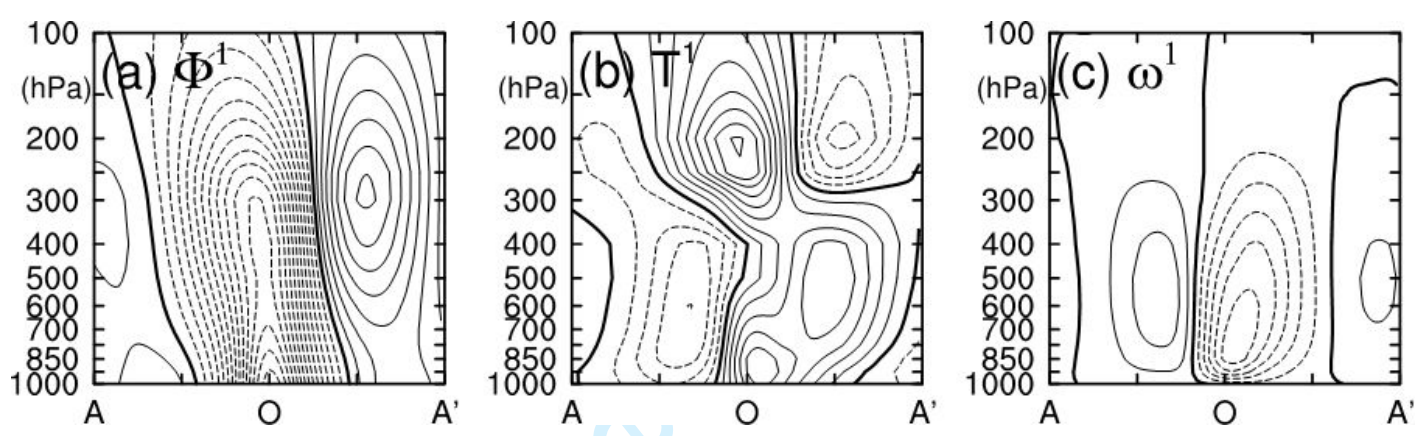

300 Figure 3. Vertical section distributions of the composites of the synoptic fields: (a) 301 geopotential anomalies (contour interval $100 \mathrm{~m}^{2} \mathrm{~s}^{-2}$ ), (b) temperature anomalies (contour 302 interval $0.5 \mathrm{~K}$ ), and (c) vertical velocity anomalies (contour interval $0.04 \mathrm{~Pa} \mathrm{~s}^{-1}$ ).

303 Negative contours are dashed and the zero contour is in bold. All these sections are taken 304 along the line $\left(A O A^{\prime}\right)$ as indicated in Figure 2i. The corresponding distributions of the 305 standard deviation are given in Figure S4 in the supplementary file.

Figures $2 \mathrm{i}-21$ show the distributions of the composite synoptic fields, which exhibit 307 more local features than the background fields. The composite field of the $500-\mathrm{hPa} \phi^{\sim 1}$ 308 shows a low-high pair (Figure 2i). The low anomaly has compact structure and large 309 amplitude, accompanying a strong cyclonic circulation (Figure 2j), whereas its 310 counterpart is broad and weak. For the other anomaly fields $\left(T^{\sim 1}\right.$ and $\left.\omega^{\sim 1}\right)$, generally 311 show a dipolar pattern (Figures $2 \mathrm{k}$ and 21). Their distributions are configured in the way 
312 that the moist-warm air ascends in the front part of the storm and the dry-cold air 313 subsides in the rear part.

314 The vertical structure of the composite synoptic system is also examined. Figure 3 315 shows the distributions of the composite synoptic fields along the section $A O A^{\prime}$ as 316 indicated in Figure 2i. This generally exhibits the structure of typical midlatitude 317 baroclinic waves, with a zonal wavelength of $\sim 4000 \mathrm{~km}$ and a westward (eastward) tilting 318 of the phase line of $\phi^{\sim 1}\left(T^{\sim 1}\right)$ with altitude (Chang, 1993; Hartmann, 1974; Lau, 1979;

319 Lim and Wallace, 1991). However, note the strong cyclone-anticyclone asymmetry. For 320 example, the cyclone is strongest at low levels, whereas the accompanying anticyclone 321 achieves its maximum at upper levels with weak signal at the earth surface (Figure $3 a) .{ }^{1}$

322 For $\omega^{\sim 1}$, the upward motion in the front part is much stronger than the downward motion 323 in the rear (Figure 3c).

324 6. Nonlinear eddy-mean flow interaction

\subsection{Spatial structure of the canonical transfers}

The eddy-mean flow interaction includes the canonical transfer from the mean flow

327 to the eddies $\left(\Gamma_{\mathrm{A}}^{0 \rightarrow 1}\right.$ and $\left.\Gamma_{\mathrm{K}}^{0 \rightarrow 1}\right)$ and for the opposite direction $\left(\Gamma_{\mathrm{A}}^{1 \rightarrow 0}\right.$ and $\left.\Gamma_{\mathrm{K}}^{1 \rightarrow 0}\right)$. The

328 former is a quantitative description of the mean-to-eddy transfer and the latter

329 characterizes the eddy feedback. We first look at the spatial structure of the former.

\footnotetext{
${ }^{1}$ To the best of our knowledge, the splitting of the warm center (at $850 \mathrm{hPa}$ ) ahead of the cyclone has not been observed (Figure 3b).
} 
(a) $\Gamma_{\mathrm{A}}^{0 \rightarrow 1}$

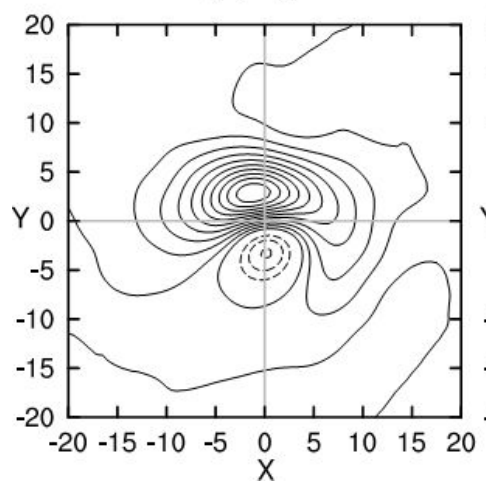

(b) $\Gamma_{\mathrm{K}}^{0 \rightarrow 1}$

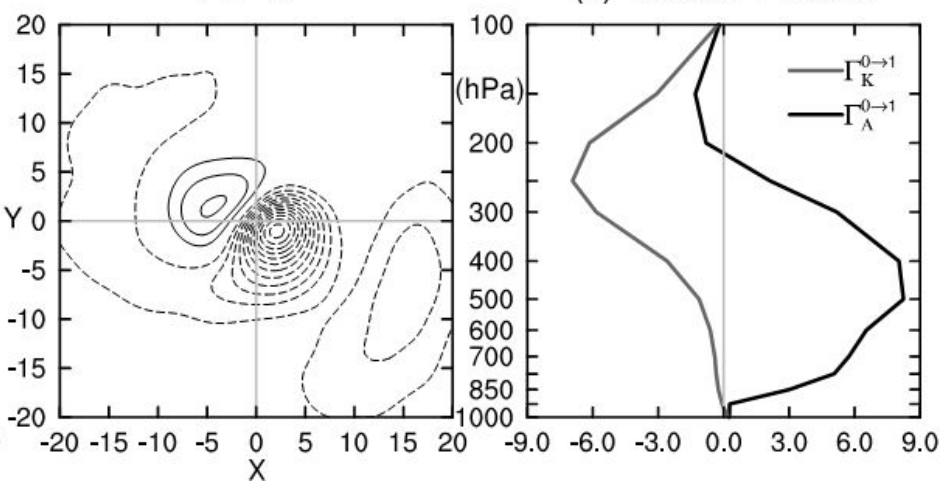

330

331

332

333

334

Figure 4. Horizontal distributions of the vertically integrated (from $1000 \mathrm{hPa}$ to $100 \mathrm{hPa}$ )

(a) $\Gamma_{A}^{0 \rightarrow 1}$ and (b) $\Gamma_{K}^{0 \rightarrow 1}$ at the maximal intensity time (t=0), with a contour interval of $2 \times 10^{-4} \mathrm{~m}^{2} \mathrm{~s}^{-3}$; and (c) the vertical distributions of $\Gamma_{A}^{0 \rightarrow 1}$ and $\Gamma_{K}^{0 \rightarrow 1}\left(10^{-4} \mathrm{~m}^{2} \mathrm{~s}^{-3}\right)$ averaged over the storm area. The corresponding distributions of the standard deviation are given in Figure S5 in the supplementary file.

Figure 4 shows the horizontal distributions of the vertically integrated (from 1000 hPa to $100 \mathrm{hPa}) \Gamma_{\mathrm{A}}^{0 \rightarrow 1}$ and $\Gamma_{\mathrm{K}}^{0 \rightarrow 1}$. Figure 4 a shows a dipolar pattern for the $\Gamma_{\mathrm{A}}^{0 \rightarrow 1}$, with a strong positive center in the north of the storm and a weak negative center in the south.

This means that the background flow experiences a downscale canonical APE transfer in the north of the storm, while an upscale canonical APE transfer in the southern region. The barotropic canonical transfer $\Gamma_{\mathrm{K}}^{0 \rightarrow 1}$ (Figure 4b), also exhibits a dipolar distribution within the storm area, with a negative region at the front and a positive one at the rear, implying upscale and downscale canonical KE transfers in the respective regions. In terms of the amplitude the negative barotropic canonical transfer center is stronger than its positive counterpart. On the whole, the storm has a favorable configuration for APE to be transferred from the mean flow, and for $\mathrm{KE}$ to be transferred to the mean flow, 
347 consistent with the traditional perspective (Barnes and Young, 1992; Orlanski and Chang, 348 1993; Simmons and Hoskins, 1978, 1980). Figure 4c shows the vertical profiles of $\Gamma_{A}^{0 \rightarrow 1}$ 349 and $\Gamma_{\mathrm{K}}^{0 \rightarrow 1}$ averaged horizontally over the storm area. We see that $\Gamma_{\mathrm{A}}^{0 \rightarrow 1}$ is positive 350 almost throughout the entire troposphere. The maximum center occurs at $400 \mathrm{hPa}$. In 351 contrast, $\Gamma_{\mathrm{K}}^{0 \rightarrow 1}$ is negative through the whole depth, implying the kinetic energy transfer 352 is from the storm to the background flow. It is strong at upper levels and reaches its 353 maximum magnitude at $250 \mathrm{hPa}$.
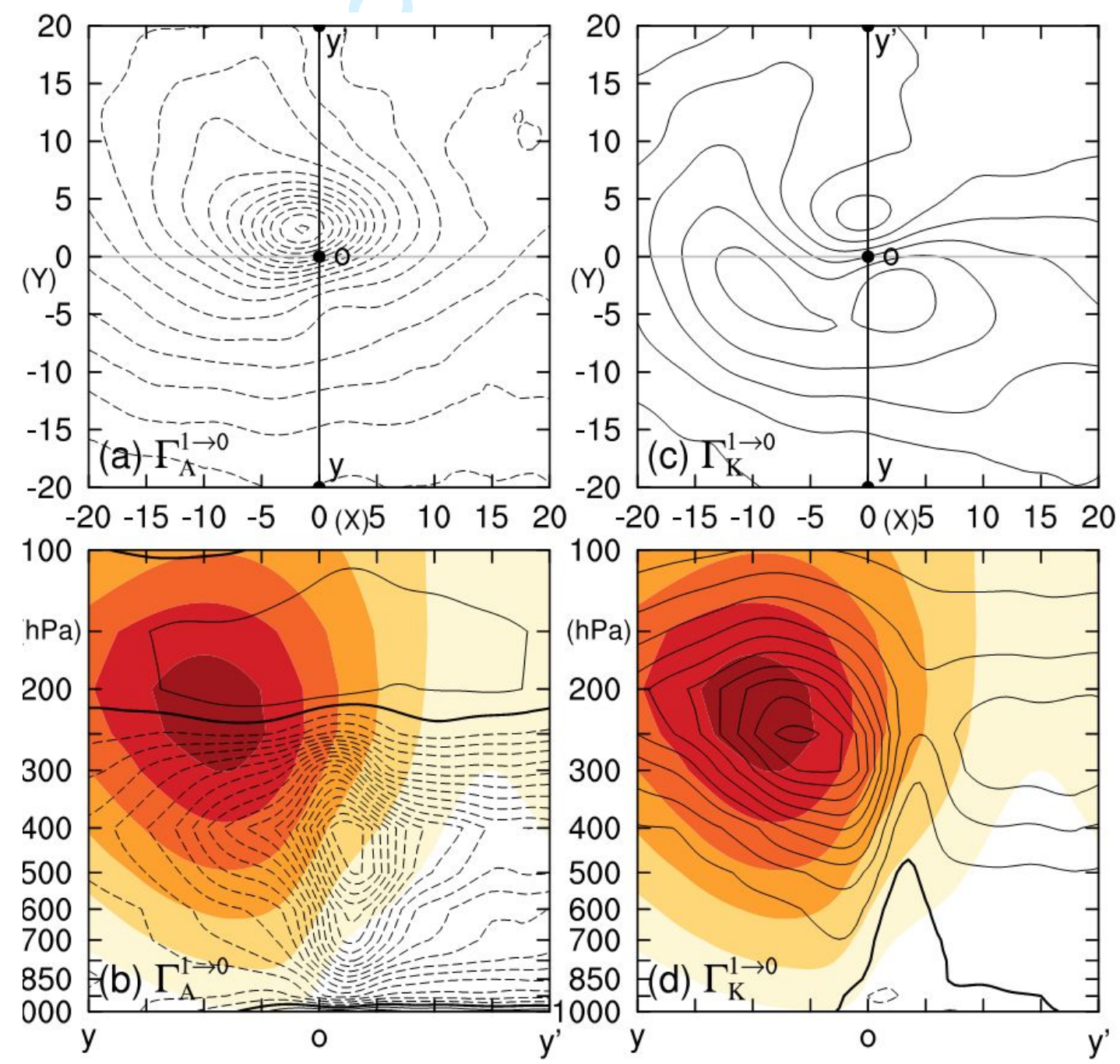

355 Figure 5. Spatial distributions of $\Gamma_{A}^{1 \rightarrow 0}$ and $\Gamma_{K}^{1 \rightarrow 0}$ at the maximal intensity time $(\mathrm{t}=0)$ : 
356 the vertically averaged (a) $\Gamma_{A}^{1 \rightarrow 0}$ and (c) $\Gamma_{K}^{1 \rightarrow 0}$; the sectional distributions of (b) $\Gamma_{A}^{1 \rightarrow 0}$ 357 and (d) $\Gamma_{K}^{1 \rightarrow 0}$ along the line yoy as indicated in (a) and (c). The contour interval is $3580.5 \times 10^{-4} \mathrm{~m}^{2} \mathrm{~s}^{-3}$. In (b) and (d), overlaid is the background wind speed $\left|\boldsymbol{v}^{\sim 0}\right|$ (shaded; 359 contour interval $4 \mathrm{~m} \mathrm{~s}^{-1}$ ). The corresponding distributions of the standard deviation are 360 given in Figure S6 in the supplementary file.

361 The spatial distributions of the eddy feedback, characterized by $\Gamma_{\mathrm{A}}^{1 \rightarrow 0}$ and $\Gamma_{\mathrm{K}}^{1 \rightarrow 0}$, are 362 shown in Figure 5. We see that horizontally $\Gamma_{\mathrm{A}}^{1 \rightarrow 0}$ is negative within the entire domain 363 (Figure 5a), implying a downscale canonical APE transfer. But, the transfer is 364 inhomogenous within the composite storm, with strong energy transfer mainly confined 365 in the northern area. Vertically, $\Gamma_{\AA}^{1 \rightarrow 0}$ is negative through almost the entire troposphere 366 (Figure 5b). The peak occurs at $400 \mathrm{hPa}$ and on the northern flank of the jet (also the 367 storm center). In short, the spatial distribution of $\Gamma_{\mathrm{A}}^{1 \rightarrow 0}$ is similar to that of $\Gamma_{\mathrm{A}}^{0 \rightarrow 1}$. For $368 \Gamma_{\mathrm{K}}^{1 \rightarrow 0}$, it is generally positive (Figure $5 \mathrm{c}$ ), implying an upscale KE canonical transfer. Note 369 that this upscale transfer is significant at upper levels, especially at the jet core (Figure $3705 \mathrm{~d})$. Figure 6 gives the vertical profiles of $\Gamma_{\mathrm{A}}^{1 \rightarrow 0}$ and $\Gamma_{\mathrm{A}}^{1 \rightarrow 0}$. It can be seen that their 371 distributions are almost the same as the mean-to-eddy counterparts (Figure 4c), but with 372 the opposite sign. 


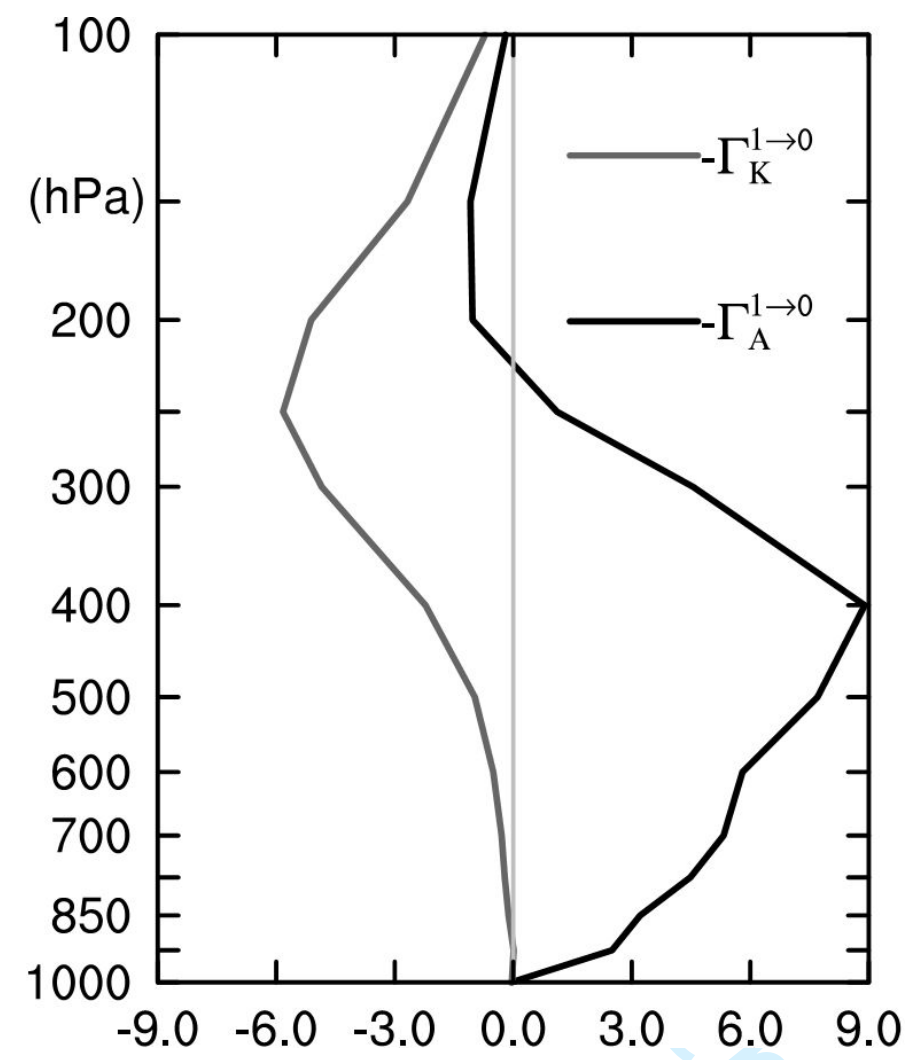

374 Figure 6. The vertical distributions of $-\Gamma_{A}^{1 \rightarrow 0}$ and $-\Gamma_{K}^{1 \rightarrow 0}\left(10^{-4} \mathrm{~m}^{2} \mathrm{~s}^{-3}\right)$ averaged over

375 the storm area.

$376 \quad 6.2$ Time evolution

377 The nonlinear eddy-mean flow interaction is not steady. Here, we are more 378 interested in how the eddy-feedback process evolves where there is a storm passing 379 through. To show this for a particular storm, we first identify the central location of the 380 storm at its maximum intensity time $(\mathrm{t}=0)$, and then sample the vertical cross section of 381 the fields (here $\Gamma_{\mathrm{A}}^{1 \rightarrow 0}$ and $\Gamma_{\mathrm{K}}^{1 \rightarrow 0}$ ) at that location meridionally at each time step from -12 382 to 12 (corresponding to from day -3 to day 3$)^{1}$. In sampling, the fields at each time step

\footnotetext{
${ }^{1}$ Here we choose a duration of 6 days, considering that the mean life cycle of the 2189 storms lasts $5.37( \pm 0.03)$ days. 
are also rotated with respect to the storm propagation direction at $\mathrm{t}=0$. Following this way, we can get the latitude-time section of any field at a particular location as a storm approaches, passes, and recedes ${ }^{1}$. This procedure is applied to each of the 2189 selected storms. Finally, the obtained sections are averaged over all the samples at each time step.
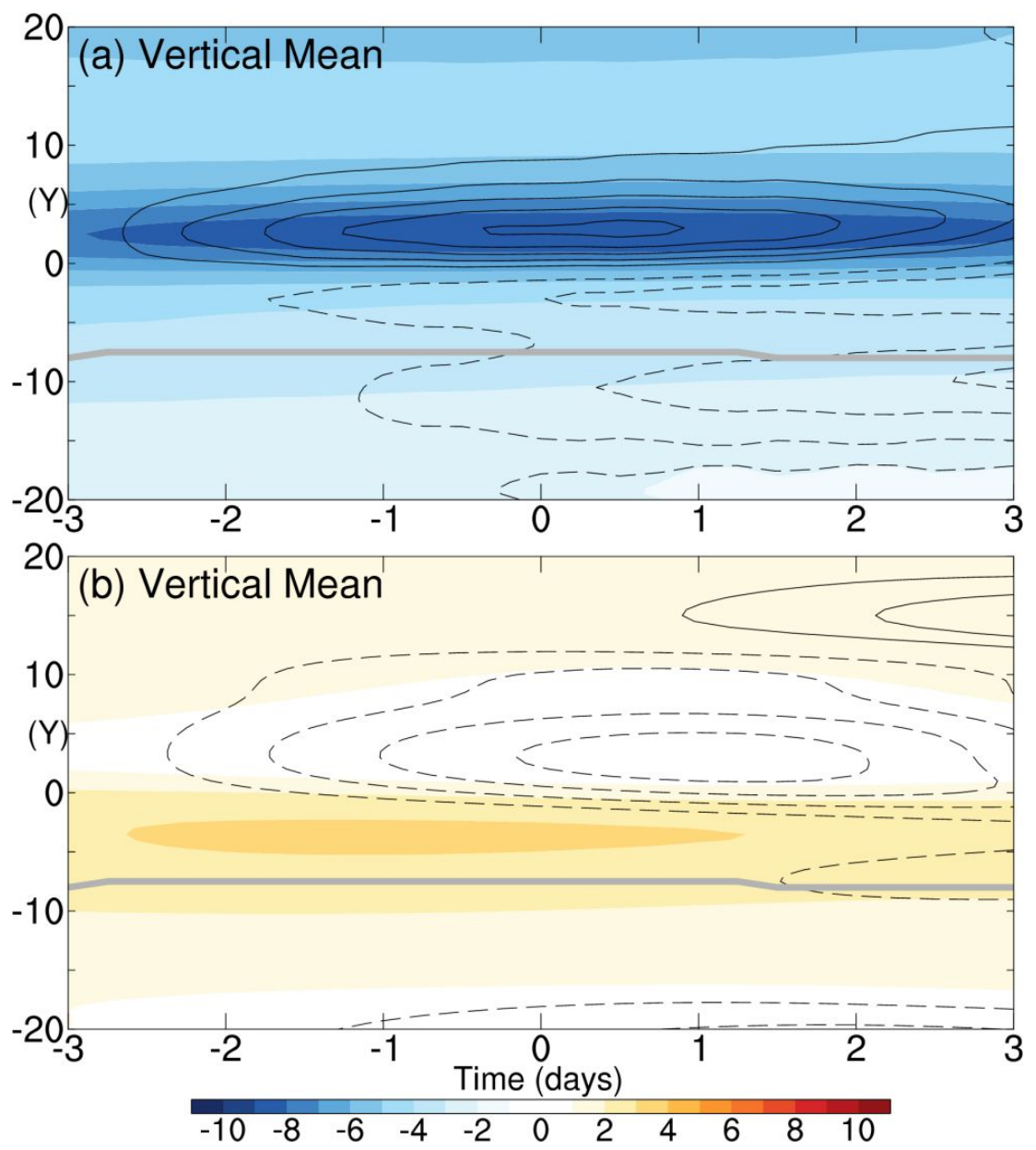

Figure 7. Vertically averaged latitude-time sections of the composite $\Gamma_{\mathrm{A}}^{1 \rightarrow 0}\left(\mathrm{a} ; 10^{-4} \mathrm{~m}^{2}\right.$

$\left.389 \mathrm{~s}^{-3}\right)$ and $\Gamma_{\mathrm{K}}^{1 \rightarrow 0}\left(\mathrm{~b} ; 10^{-4} \mathrm{~m}^{2} \mathrm{~s}^{-3}\right)$. The contours show the changes in magnitude relative to 390 the initial state (at day -3 ), which are calculated as $A^{\prime}=|A|-\left|A_{*}\right|$, with $A$ the original

\footnotetext{
${ }^{1}$ Here we switch to the Eulerian approach in order to obtain the spatio-temporal distribution of the interaction and, particularly, the background flow response (see below).
} 
391 field and $A_{*}$ the field at day $-3^{1}$. The contour interval is $0.2 \times 10^{-4} \mathrm{~m}^{-2} \mathrm{~s}^{-3}$. The thick

392 gray curve in (a) and (b) denotes the latitude where the $300-\mathrm{hPa}$ background wind is 393 maximized (i.e., jet axis). The corresponding distributions of the standard deviation are 394 given in Figure S7 in the supplementary file.

Figure 7 shows the general time evolution of $\Gamma_{\mathrm{A}}^{1 \rightarrow 0}$ and $\Gamma_{\mathrm{K}}^{1 \rightarrow 0}$. We see that as the 396 storm passes, the downscale APE transfer is intensified within a narrow zonal band north 397 of the storm center, whereas elsewhere it is slightly weakened (Figure 7a). In contrast, the 398 upscale KE transfer is intensified slightly within a narrow zonal band on the southern 399 side, while in the north it is greatly weakened (Figure $7 \mathrm{~b}$ ). The changes of $\Gamma_{\mathrm{A}}^{1 \rightarrow 0}$ and $400 \quad \Gamma_{\mathrm{K}}^{1 \rightarrow 0}$ begin from about 3 days before the storm arrives, achieve their maxima as the 401 storm arrives, and then weaken. In the presence of the storm, $\Gamma_{\mathrm{A}}^{1 \rightarrow 0}$ and $\Gamma_{\mathrm{K}}^{1 \rightarrow 0}$ seem to 402 take place complementarily in the horizontal plane: $\Gamma_{\mathrm{K}}^{1 \rightarrow 0}$ intensifies (weakens) wherever $403 \quad \Gamma_{\mathrm{A}}^{1 \rightarrow 0}$ substantially weakens (intensifies).

404 The most interesting finding in this section is the spatial asymmetry of the 405 eddy-mean flow interaction: the downscale canonical APE transfer process mainly 406 happens in the northern part of the storm (also on the northern flank of the jet stream) and 407 in middle levels, whereas the upscale canonical KE transfer mainly occurs in the south, 408 overlaid with the jet core. The former acts to destroy the background baroclinicity, 409 whereas the latter acts to accelerate the jet and thus enhance the baroclinicity. These

\footnotetext{
${ }^{1}$ Here we want to see the change in energy transfer as a storm arrives but initially there is no storm, so we choose an earlier day (i.e., day -3 ) rather than day 0.
} 
410 competing effects essentially determine the final state of the jet stream after the storm

411 passes. We will elaborate on this in the following section.

412 7. Response of the background flow and baroclinicity

413 In this study, we focus on the responses of the background wind and baroclinicity.

414 Figure 8 shows the composite sections of the background wind (left column). We see that 415 as the storm passes, the wind decreases in the north, whereas it increases in the south, 416 showing an anomalous dipolar pattern (Figure 8a). Moreover, the increase in the south is 417 stronger than the decrease in the north. Since the jet core is located in the south of the 418 storm (cf. Figure 3), this tends to strengthen and narrow the jet (by 2 latitudes) ${ }^{1}$. Figures $4198 \mathrm{c}$ and $8 \mathrm{~d}$ show the composite pressure-time sections of the background wind along the 420 latitudes of $10^{\circ}$ and $-10^{\circ}$, respectively. It can be seen that either in the north or in the 421 south, the response of the background wind is more significant in the upper troposphere 422 (Figures 8b and 8c). Temporally, the background wind begins to change from about 3 423 days before the storm's arrival, and responds strongly as the storm arrives. A closer 424 observation reveals that the largest change occurs at $t=2 d$ instead of $t=0$ (see also the 425 energy transfers in Figure 7). This may be due to the consecutive influence of secondary 426 cyclones formed along the trailing cold front of the reference one (e.g., Papritz and 427 Schemm 2013). Overall, the jet does not show any obvious meridional shift. This is 428 consistent with the argument of Vallis and Gerber (2008) that, if the storm is generated

\footnotetext{
${ }^{1}$ This estimation is based on the half jet-width, which is measured by the distance between the latitude of maximum wind and the northward latitude at which the wind reaches half of its peak. 
on the flank of the jet, the storm would act to maintain the jet's latitude. For the

430 background wind response at any instant (e.g., $\mathrm{t}=0$ ), the meridional structure is similar to

431 that obtained by Chang (2001b) using wave packet regression.
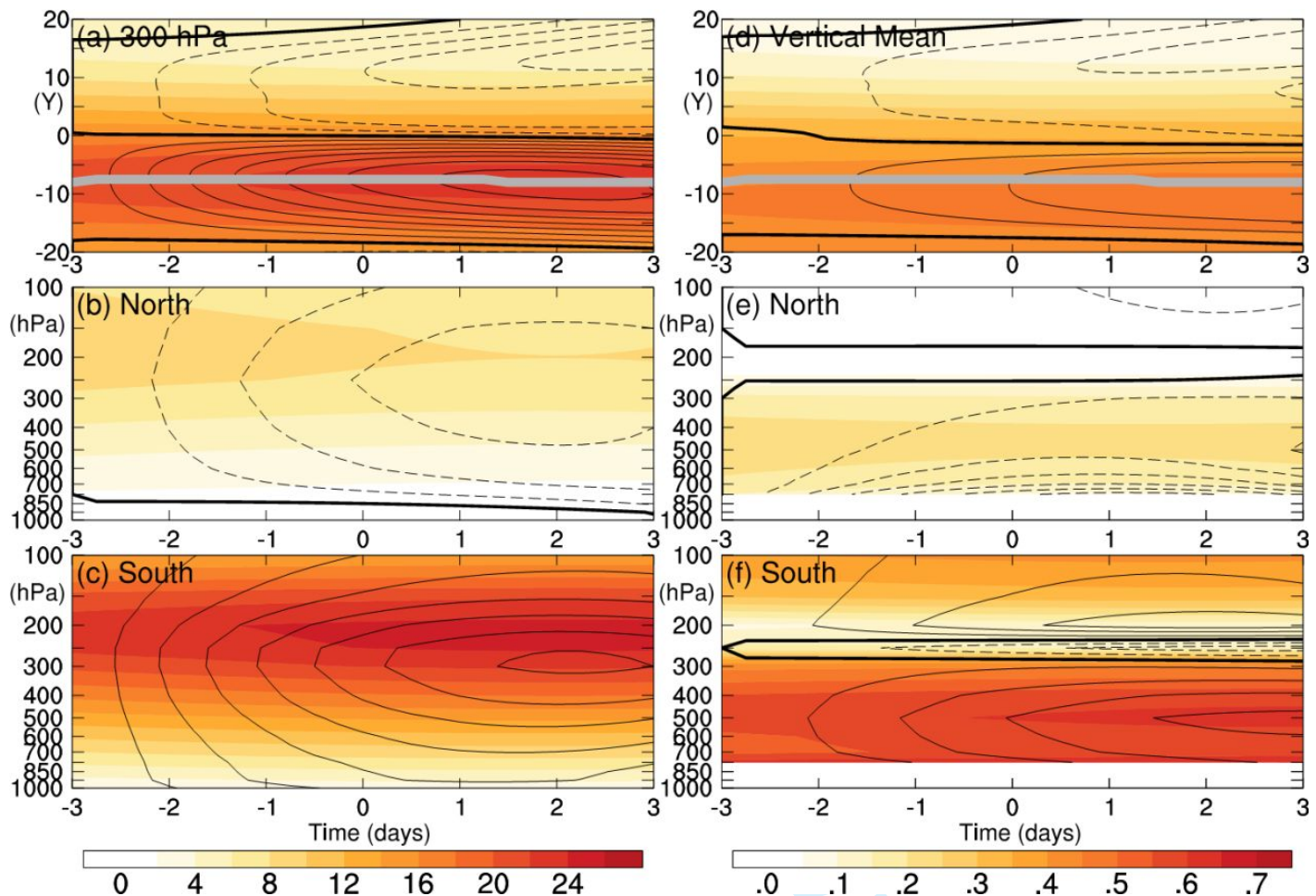

433 Figure 8. Left column: same as Figure 7, but for the background wind speed (shaded, $\mathrm{m}$

$434 \mathrm{~s}^{-1}$ ) and its anomaly (contoured, with an interval of $0.25 \mathrm{~m} \mathrm{~s}^{-1}$ ); (a) shows the

435 latitude-time section at $300 \mathrm{hPa}$; (b) and (c) show the pressure-time sections along the 436 latitudes of $10^{\circ}$ and $-10^{\circ}$, respectively. The thick gray curve in (a) and (d) denotes the

437 latitude of the maximum of the 300-hPa background wind. Right column: same as the left 438 column, but for the baroclinicty, i.e., the Eady growth rate (shaded; in day $^{-1}$ ) and its 439 anomaly (contoured, with an interval of 0.01 day $^{-1}$ ). The corresponding distributions of 440 the standard deviation are given in Figure S8 in the supplementary file. 
442 is the static stability and other symbols are conventional), is also shown in Figure 8 (right

443 column). We see that, as the storm passes, the baroclinicity weakens in the north, whereas

444 it strengthens in the south (Figure 8d). Vertically, the largest northern weakening happens

445 in the lower troposphere (Figure 8e), while the strongest southern strengthening occurs at 446 middle levels (Figure 8f).

447 There is a discrepancy between the evolution of the energy transfers and that of the 448 background flow. Figure 7 suggests that the downscale transfer north of the cyclone is 449 much stronger than the upscale transfer south of the cyclone, while Figure 8 show that the $450 \mathrm{jet} /$ baroclinicity decrease north of the cyclone is somewhat smaller than the jet/baroclinic 451 increase south of the cyclone. How does this discrepancy happen? In fact, the energy 452 transfer is not the only factor that can cause change in the background field. In addition to 453 the nonlinear interaction process, many other energy processes (e.g., energy flux 454 convergences) are also involved, and they can redistribute the energy in horizontal space 455 (Papritz and Schemm 2013; Rivière et al. 2015). Figure 9 shows the mean distribution of 456 the horizontal kinetic energy flux convergence along the meridional direction. It can be 457 seen that the convergence is strong on the southern side of the storm center and peaks 458 near the jet center. Moreover, the convergence is mainly due to the zonal component of 459 the KE flux (Figure 9b). This indicates that, apart from the energy transfer, nonlocal 460 processes also play a role in the enhancement of the background flow in the southern side 461 of the storm. 


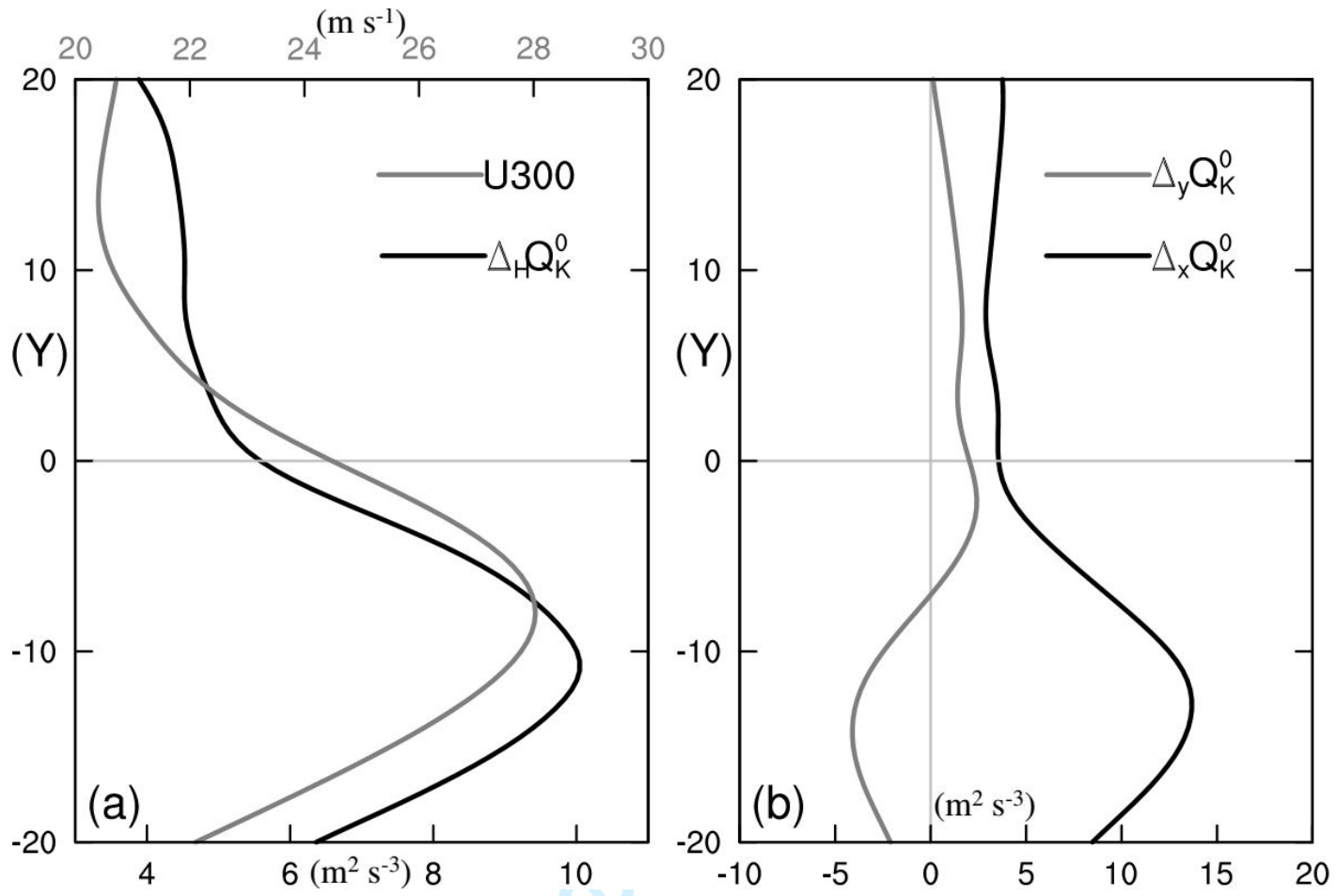

Figure 9. (a) Mean distributions of the horizontal kinetic energy flux convergence (in

black; $10^{-4} \mathrm{~m}^{2} \mathrm{~s}^{-3}$ ) and 300-hPa wind speed (in gray; $\mathrm{m} \mathrm{s}^{-1}$ ) versus $y$; (b) same as (a), but for the $\mathrm{x}$ - and $\mathrm{y}$-components of the kinetic energy flux convergence.

\section{Discussion}

467

In the above we have discussed about the average results of all the 2189 storms, and 468 overall the composite storm is located on the northern side of the maximum background

469 wind speed (refer to Figures 2, 5, etc.). But if we have a closer look at these storms, 470 actually four types of relative locations can be classified.

In order to determine the relative location of a storm, we sample the $300-\mathrm{hPa}$ zonal

472 wind in equal-distance along the meridian passing through the storm center. We take the

473 center as the starting point $\left(0^{\circ}\right)$ and sample the wind speed once every $2.5^{\circ}$ to the south 474 and north, respectively. The sampling range is from $-20^{\circ}$ (south) to $20^{\circ}$ (north). Based on 
475 the resulting meridional profile of the background zonal wind, the relative location is then 476 determined. We first calculate the average meridional gradient of zonal wind north and 477 south of the storm center, which here is simply represented by the difference $\Delta=$ $478 \overline{u_{\mathrm{i}+1}-u_{\mathrm{i}}}$ (the overbar stands for meridional average). For convenience, the average 479 gradient on the southern side and the northern side are referred to as $\Delta_{S}$ and $\Delta_{N}$, 480 respectively. If $\Delta_{S}<0$ and $\Delta_{N}<0$, it means that the storm center is located on the 481 northern side of the jet, and we call this type of configuration Type-1; if $\Delta_{S}>0$ and $\Delta_{N}$ $482>0$, the storm center is located on the southern side of the jet, and we call this 483 configuration Type-2; if $\Delta_{S}>0$ and $\Delta_{N}<0$, the storm is at the center of the jet, and the 484 configuration is classified as Type-3; if $\Delta_{S}<0$ and $\Delta_{N}>0$, it means that there are two 485 jets, and the storm lies in between---we call this configuration Type-4.

486 By the above classification it is found that there are 909 Type-1, 93 Type-2, 881 487 Type-3, and 299 Type-4 configurations, plus 7 cases belonging to nowhere. The mean 488 meridional profiles of the background zonal wind corresponding to these four 489 configuration types are shown in Figure 10. It can be seen that if only the configuration 490 between the storm and the major jet is considered, the Type- 4 case is similar to the 491 Type-1 case, i.e., the configuration with storms located on the northern side of the major 492 jet. The result is, the Type- 1 and the Type- 4 storms together account for $55.2 \%$ of the 493 total 2189 storms, consistent with the previous observation that the storm track is 494 generally located on the poleward flank of the jet (e.g., Blackmon et al., 1977; Chang et 495 al., 2002). On the other hand, the Type-3 storms are not exactly located at the center of 
496 the jet, but northward of it by $2.5^{\circ}$. Despite being a minority, 93 storms appear southern 497 side of the jet. One may wonder whether these four kinds of storms have different 498 interaction scenarios from each other. In the following, we discuss these four types of 499 storms separately and compare and contrast them.

(a) Type-1

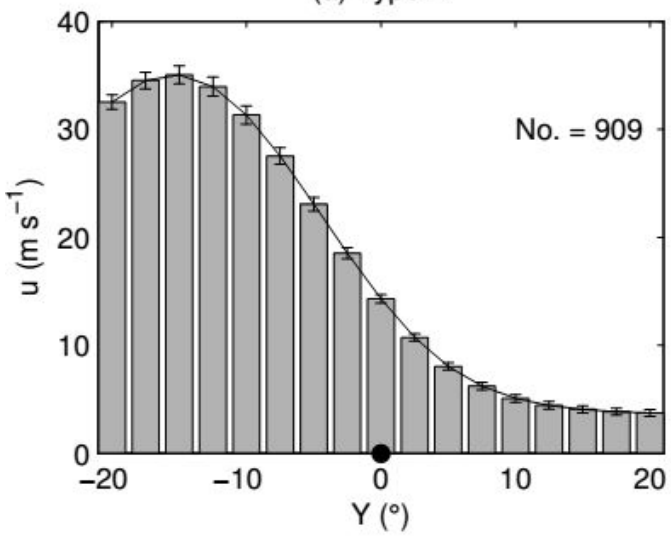

(c) Type-3

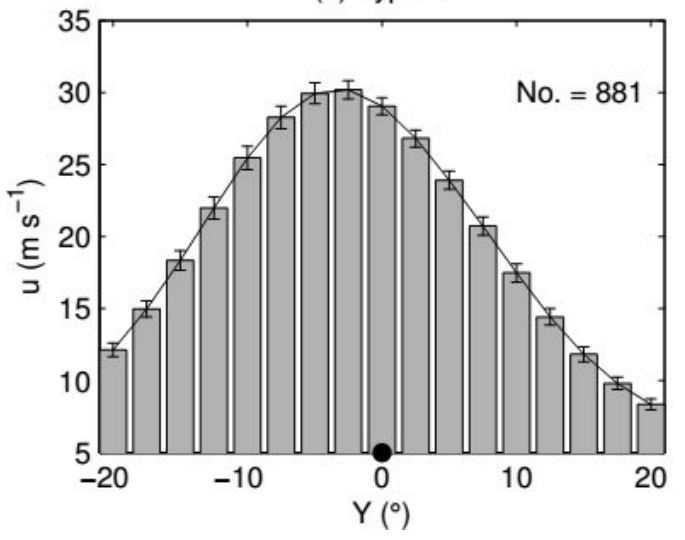

(b) Type-2

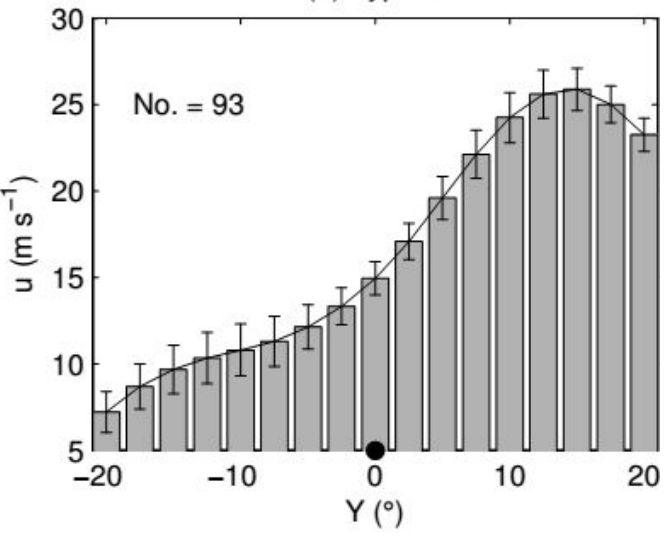

(d) Type-4

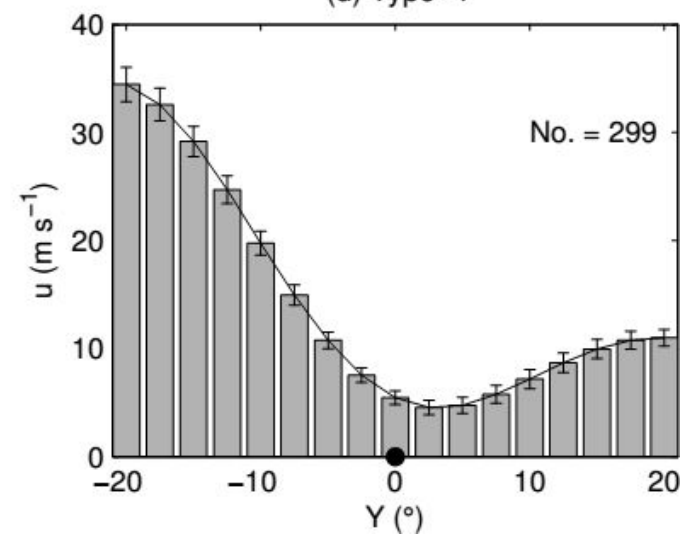

500

501 Figure 10. The four types of zonal wind profiles. (a) Type-1: the storm is on the northern

502 side of the jet; (b) Type-2: the storm is on the southern side of the jet; (c) Type-3: the

503 storm is at the jet center; and (d) Type-4: the storm is located between two jets. The black

504 dot indicates the meridional location of the storm center. The error bars represent the 505 uncertainties of the mean at the 5\% significance level.

506 Figure 11 shows the distribution of the composite $500-\mathrm{hPa}$ geopotential anomalies 
507 for the four types of storms. As expected, the distributions of the Type- 1 and Type- 4 508 storms are similar since both of them are located on the northern flank of the jet. Besides, 509 their distributions (Figures 11a-c, 11j-1) are similar to the general results as shown in 510 Figures 2a, 2e, and 2i, with a wide trough in the background field (Figures 11b, k) and a 511 dipole in the synoptic field (Figures 11c, 1). This is easy to understand because these two 512 types together account for the largest proportion of the total storms. Although the Type-3

513 storm is located near the jet center, the distribution of its geopotential anomalies (Figures

514 11g-i) is still similar to those of Type 1 and Type 4. The obvious difference lies in the 515 Type-2 storm. There is a distinct anti-cyclonic circulation on the southern side of the 516 storm in the original field (Figure 11d), which is not seen in the other three types. In 517 addition, it can be found that the propagation direction of the Type-2 storm is basically 518 parallel to the isolines of the background geopotential (Figure 11e), whereas that of the 519 other three types intersects the isolines and points to the left side. 


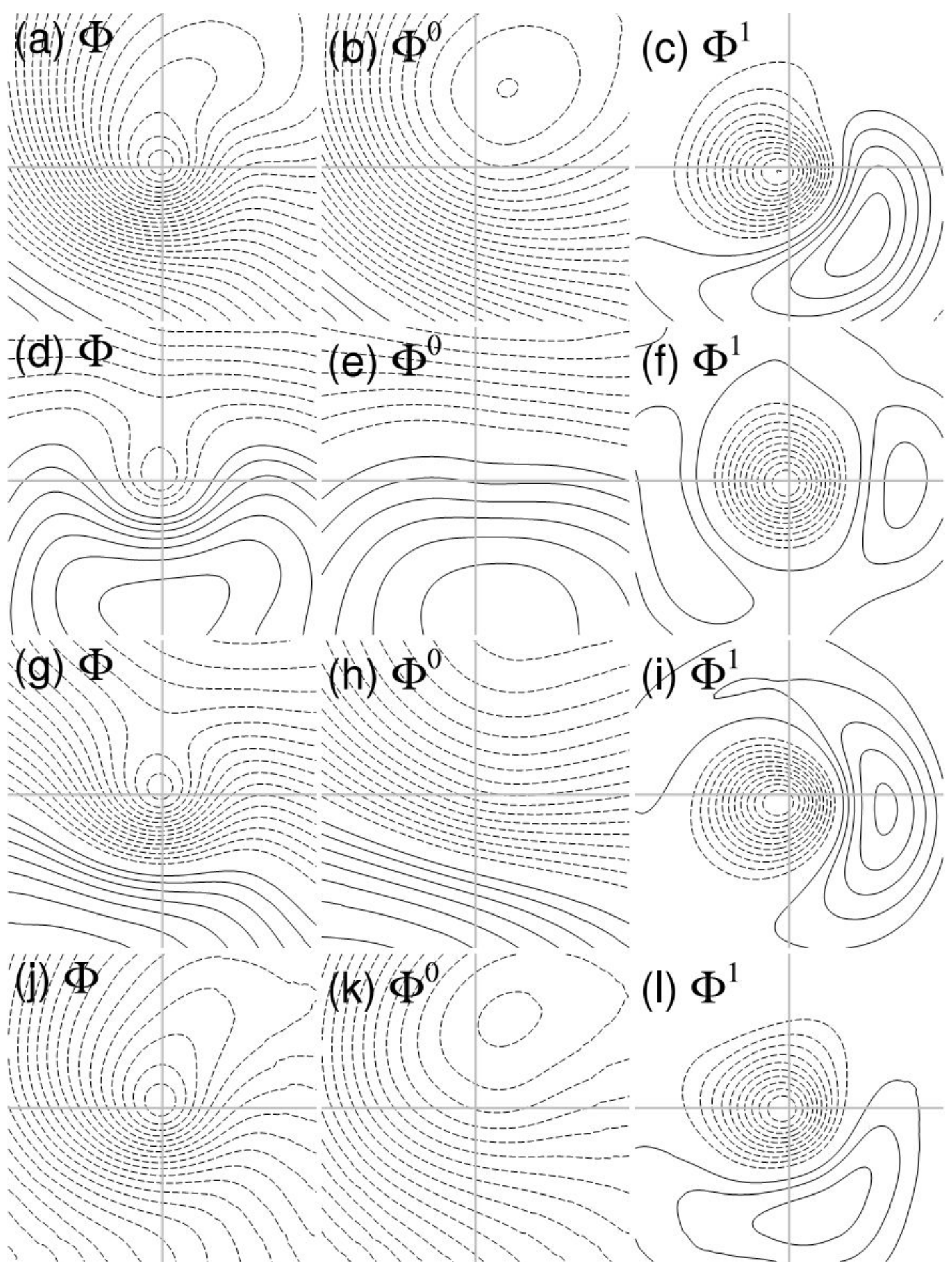

521 Figure 11. Same as Figures 2a, 2e, and 2i, but for the Type-1 storm (a, b, c), Type-2

522 storm (d, e, f), Type-3 storm ( $g$, h, i), and Type-4 storm (j, k, l), respectively. The

523 corresponding distributions of the standard deviations are displayed in Figure S9 in the 
524 supplementary file.
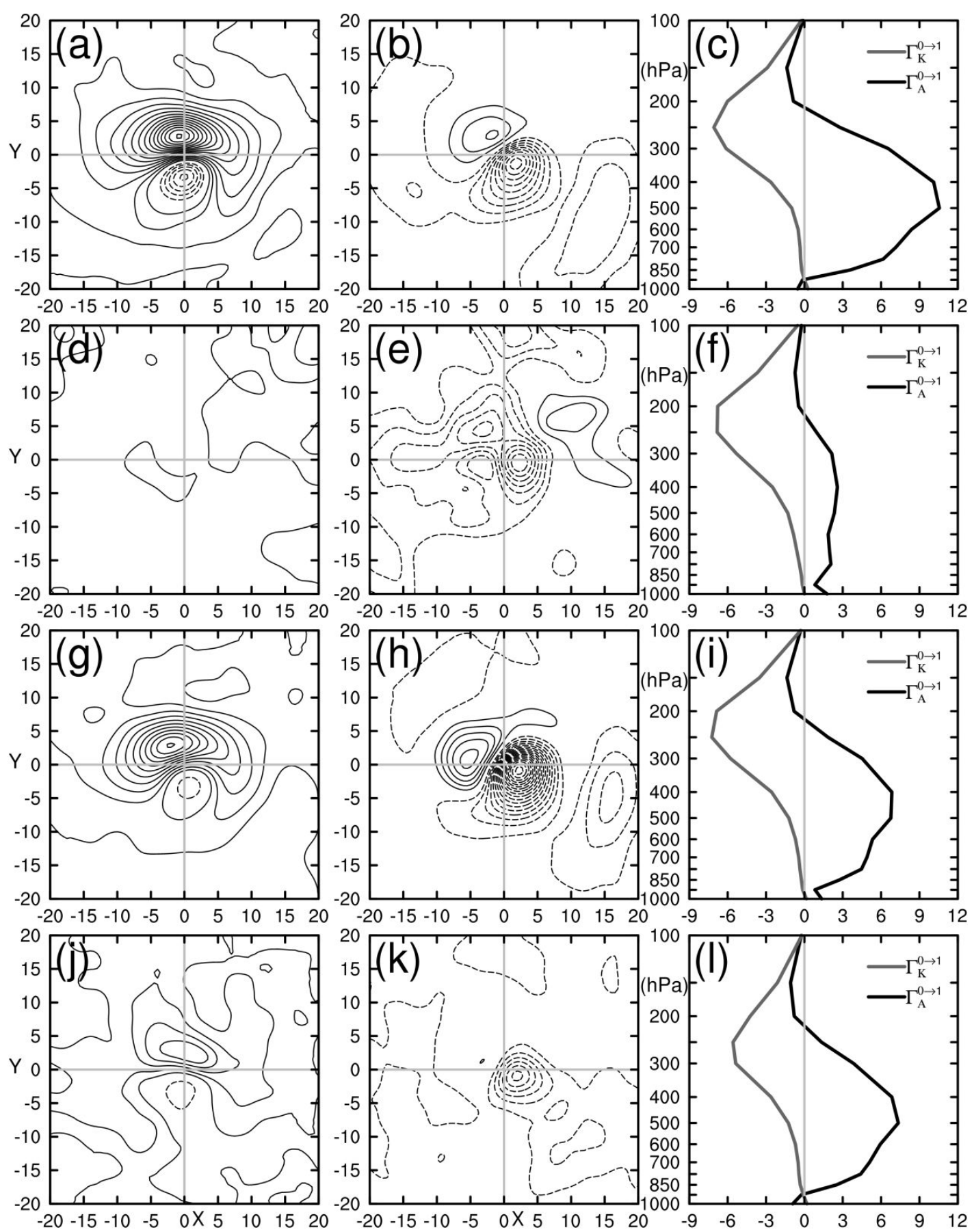

526 Figure 12. Same as Figure 4, but for the Type-1 storm (a, b, c), Type-2 storm (d, e, f),

527 Type-3 storm (g, h, i), and Type-4 storm (j, k, 1), respectively. Left column: $\Gamma_{\mathrm{A}}^{0 \rightarrow 1}$;

528 Middle column: $\Gamma_{\mathrm{K}}^{0 \rightarrow 1}$. The corresponding distributions of the standard deviations are 
529 given in Figure S10 in the supplementary file.

530

531 The horizontal distributions of the vertically integrated canonical transfers $\Gamma_{\mathrm{A}}^{0 \rightarrow 1}$

532 and $\Gamma_{\mathrm{K}}^{0 \rightarrow 1}$ are given in Figure 12. It can be seen that the Type-1 (Figures 12a, b), Type-3

533 (Figures 12g, h), and Type-4 (Figures 12j, k) storms generally bear the same structure,

534 similar to the general results as shown in Figure 4. For instance, $\Gamma_{\mathrm{A}}^{0 \rightarrow 1}$ shows a dipolar

535 pattern, with a strong positive center in the north of the storm and a weak negative center

536 in the south. $\Gamma_{\mathrm{K}}^{0 \rightarrow 1}$ also exhibits a dipolar distribution within the storm area, with a

537 negative center at the front and a positive one in the rear (note the positive $\Gamma_{\mathrm{K}}^{0 \rightarrow 1}$ center

538 in the Type-4 storm is not obvious). In contrast, the Type-2 storm is quite different.

539 Horizontally, $\Gamma_{\mathrm{A}}^{0 \rightarrow 1}$ is mainly distributed in the northeast corner with small values

540 (Figure 12d), and there is no dipole pattern as observed in the other three types. $\Gamma_{\mathrm{K}}^{0 \rightarrow 1}$

541 does not show the dipolar structure, either (Figure 12e); it is negative throughout the

542 domain, with two minima around the storm center.

543 Also presented in Figure 12 are the average vertical distributions of $\Gamma_{\mathrm{A}}^{0 \rightarrow 1}$ and

$544 \Gamma_{\mathrm{K}}^{0 \rightarrow 1}$. The Type-1 (Figure 12c), Type-3 (Figure 12i) and Type-4 (Figure 121) storms have

545 the largest amplitude of $\Gamma_{\mathrm{A}}^{0 \rightarrow 1}\left(\Gamma_{\mathrm{K}}^{0 \rightarrow 1}\right)$ at middle (high) levels, similar to the general

546 results previously revealed in Figure 4c. For the Type-2 storm, its $\Gamma_{\mathrm{K}}^{0 \rightarrow 1}$ profile is also

547 similar, but its $\Gamma_{\mathrm{A}}^{0 \rightarrow 1}$ profile is quite different. Although it is still positive through most

548 of the troposphere, it is quite weak; refer to Table 1 for a quantitative comparison.

549 
550 Table 1 The average $\Gamma_{\mathrm{A}}^{0 \rightarrow 1}$ and $\Gamma_{\mathrm{K}}^{0 \rightarrow 1}$ for the four types of storms and the corresponding 551 absolute ratio $\left|\Gamma_{\mathrm{A}}^{0 \rightarrow 1} / \Gamma_{\mathrm{K}}^{0 \rightarrow 1}\right|$.

\begin{tabular}{l|l|l|l|l}
\hline & Type-1 & Type-2 & Type-3 & Type-4 \\
\hline$\Gamma_{\mathrm{A}}^{0 \rightarrow 1}\left(10^{-4} \mathrm{~m}^{2} \mathrm{~s}^{-3}\right)$ & 5.3 & 1.5 & 3.7 & 3.5 \\
\hline$\Gamma_{\mathrm{K}}^{0 \rightarrow 1}\left(10^{-4} \mathrm{~m}^{2} \mathrm{~s}^{-3}\right)$ & -1.9 & -2.1 & -2.1 & -1.7 \\
\hline$\left|\Gamma_{\mathrm{A}}^{0 \rightarrow 1} / \Gamma_{\mathrm{K}}^{0 \rightarrow 1}\right|$ & 2.7 & 0.7 & 1.8 & 2.0 \\
\hline
\end{tabular}

552 

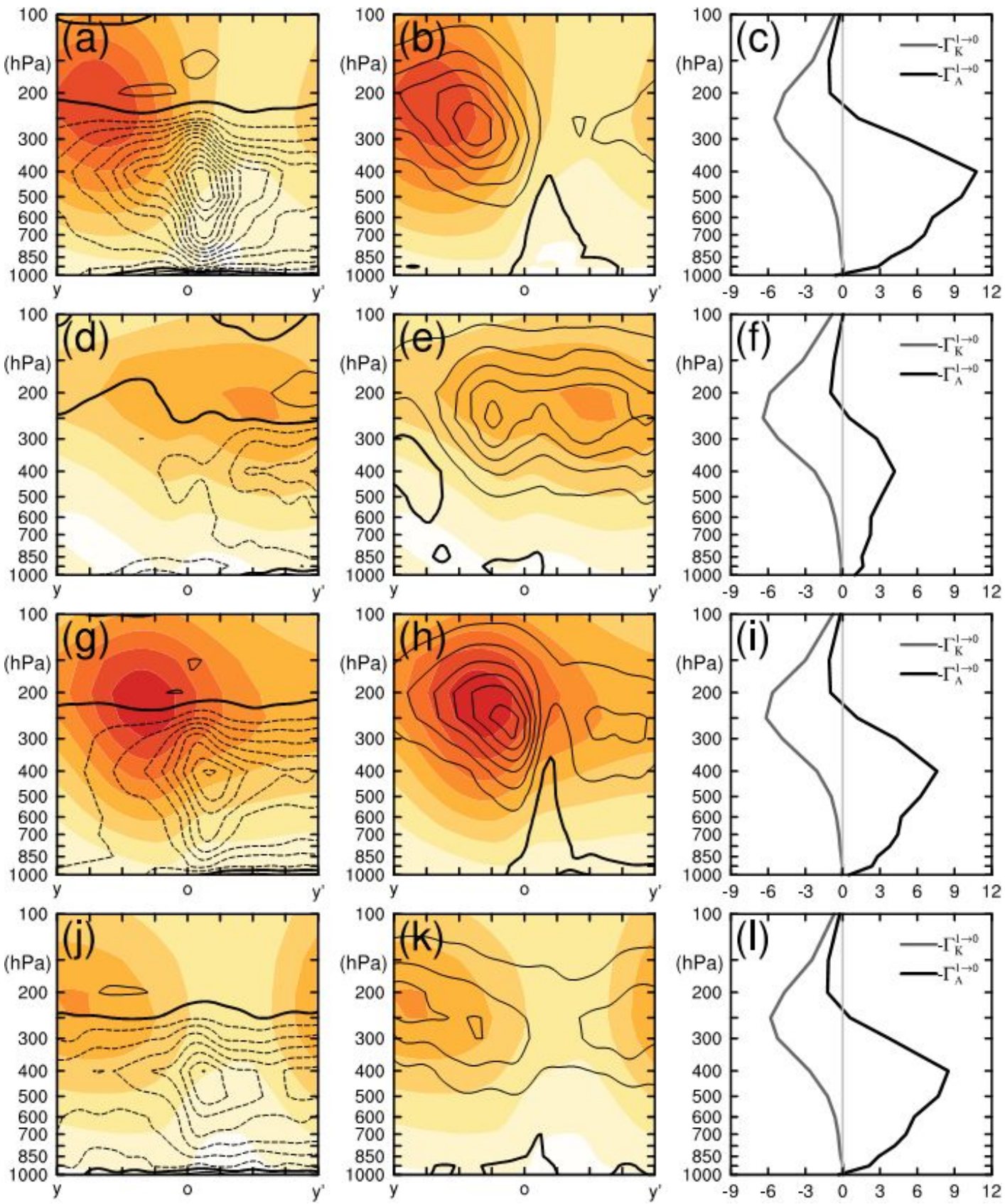

Figure 13. Same as Figures 5 and 6, but for the Type-1 storm (a, b, c), Type-2 storm (d, e,

555 f), Type-3 storm (g, h, i), and Type-4 storm (j, k, 1), respectively. Left column: $\Gamma_{A}^{1 \rightarrow 0}$;

556 Middle column: $\Gamma_{\mathrm{K}}^{1 \rightarrow 0}$. The corresponding distributions of the standard deviations are

557 given in Figure S11 in the supplementary file.

558

Figure 13 gives the distributions of $\Gamma_{A}^{1 \rightarrow 0}$ and $\Gamma_{K}^{1 \rightarrow 0}$ over the meridional section 36 
559 through the storm center. For $\Gamma_{A}^{1 \rightarrow 0}$, there is always an active center on the northern side 560 of the Type-1 (Figure 13a), Type-3 (Figure 13g), and Type-4 (Figure 13j) storms (also 561 the jets), whereas it is lacked in the Type-2 storm (Figure $13 \mathrm{~d}$ ). For $\Gamma_{K}^{1 \rightarrow 0}$, its center 562 generally coincides with the jet stream. In addition, $\Gamma_{K}^{1 \rightarrow 0}$ seems to be enhanced in the 563 southern part of the storm. For instance, the $\Gamma_{K}^{1 \rightarrow 0}$ maximum always occurs there, 564 although the jet sometime is located in the north (see Figure 13e). Vertically, the 565 distributions of $\Gamma_{A}^{1 \rightarrow 0}$ and $\Gamma_{K}^{1 \rightarrow 0}$ (Figures 13c, f, i, 1) are basically the same as the 566 mean-to-eddy counterparts (Figures 12c, f, i, 1), but with the opposite sign.

567 The time evolution of the vertically averaged $\Gamma_{A}^{1 \rightarrow 0}$ and $\Gamma_{K}^{1 \rightarrow 0}$ is given in Figure 14.

568 Generally, the evolutions of Type 1, Type 3, and Type 4 are similar, with $\Gamma_{A}^{1 \rightarrow 0}\left(\Gamma_{K}^{1 \rightarrow 0}\right)$ 569 strengthened in the north (south) of the storm and weakened in the south (north). In 570 contrast, Type 2 is different. Its $\Gamma_{A}^{1 \rightarrow 0}$ is strengthened near the storm center and is 571 weakened in the south and north (Figure 14c), whereas the $\Gamma_{K}^{1 \rightarrow 0}$ changes in the opposite 572 direction (Figure 14d). 

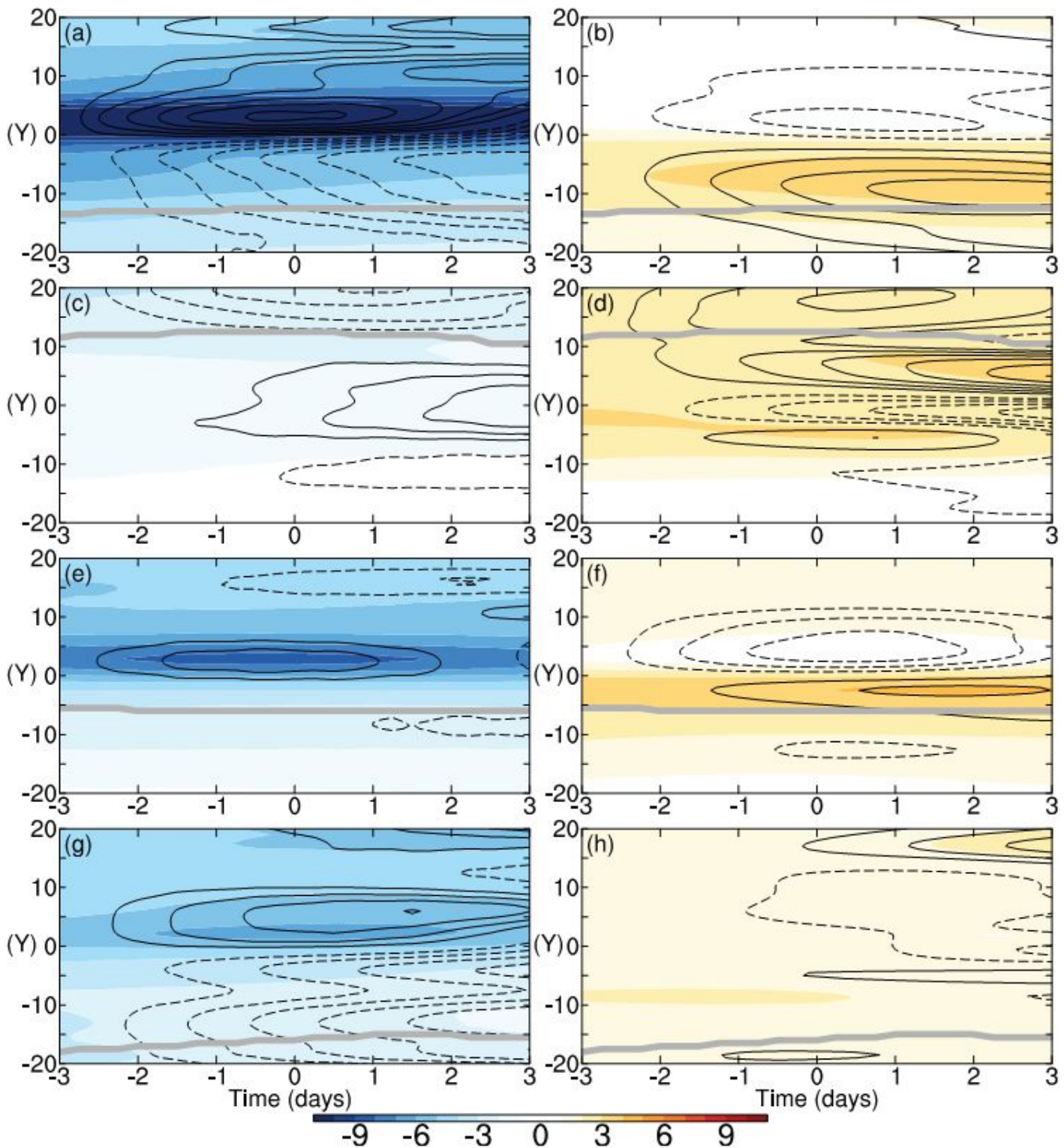

574 Figure 14. Same as Figs. 7a and 7b, but for the Type-1 storm (a, b), Type-2 storm (c, d),

575 Type-3 storm (e, f), and Type-4 storm (g, h). Left column: $\Gamma_{\mathrm{A}}^{1 \rightarrow 0}$; right column: $\Gamma_{\mathrm{K}}^{1 \rightarrow 0}$.

576 The thick gray curve denotes the latitude of the maximum of the $300-\mathrm{hPa}$ background

577 wind. The corresponding distributions of the standard deviations are given in Figure S12

578 in the supplementary file.

579

To show the response of the background wind, displayed in Figure 15 is the time 
580 evolution of the background wind speed at the lower and upper tropospheric levels.

581 Under the influence of the storm (of any type), the background wind speed is weakened

582 in the north of the storm and is strengthened in the south, showing an anomalous dipolar

583 distribution. Besides, the background wind response basically shows an equivalent

584 barotropic structure in the vertical direction, and the response is most prominent at upper

585 levels. It is worth noting that the Type-1, Type-3, and Type-4 storms are located near and

586 to the north of the jet center, thus accelerating the jet, whereas the Type- 2 storm is

587 located on the south side of the jet center, weakening the jet. In addition, the wind

588 response to the Type-2 storm is more complex than the other three types (see Figures 15c,

589 d). In general, the response of the background wind speed is consistent with that of the

590 canonical transfers, except for Type 2, implying that other processes may also play a role

591 for this type of storms (refer to sec. 7). 

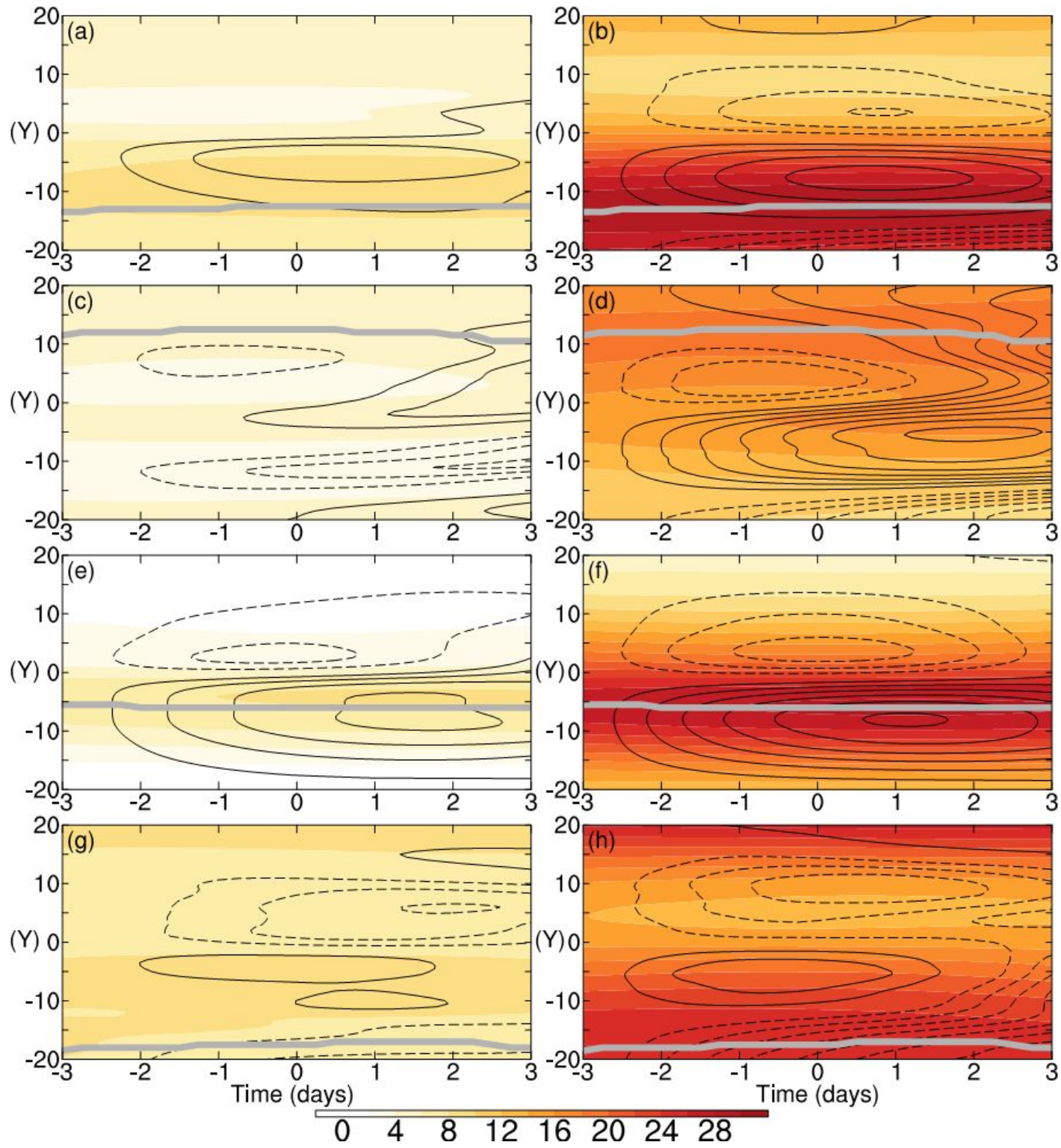

593 Figure 15. Same as Fig. 8a, but for the Type-1 storm (a, b), Type-2 storm (c, d), Type-3

594 storm (e, f), and Type-4 storm (g, h), respectively. Left column: $850 \mathrm{hPa}$; Right column:

$595300 \mathrm{hPa}$. The thick gray curve denotes the latitude of the maximum of the $300-\mathrm{hPa}$

596 background wind. The corresponding distributions of the standard deviations are given in

597 Figure S13 in the supplementary file.

598

To summarize, all the four types of storms basically exhibit a north-south 
599 asymmetry in the interaction with the background flow, especially when the storm 600 appears near or to the north of the jet center. When the storm lies to the south of the jet, 601 the baroclinic canonical transfer turns out to be weak and its overall transfer is also 602 weakened, quite different from the other three types. The response of the background 603 wind field to the four types of storms generally exhibits a north-south oriented dipolar 604 distribution, with positive anomaly in the south and negative anomaly in the north. But 605 the Type-1, Type-3, and Type-4 storms strengthen the jet stream ${ }^{1}$, whereas the Type-2 606 storm weakens the jet stream.

607 In a word, the interaction and background wind response for the Type-1, Type-3, 608 and Type-4 storms are essentially similar to what we have obtained previously with all 609 the storms considered together. The discrepancy is with the Type-2 storm. However, it 610 should be noted that here the sample for this type is too small (with only 93 members). Its 611 contribution is hence relatively limited and, besides, the reliability of the result also needs 612 to be further verified.

\footnotetext{
${ }^{1}$ For the Type-4 storm, the strengthening of the background wind at upper level happens on the northern flank of the jet. 


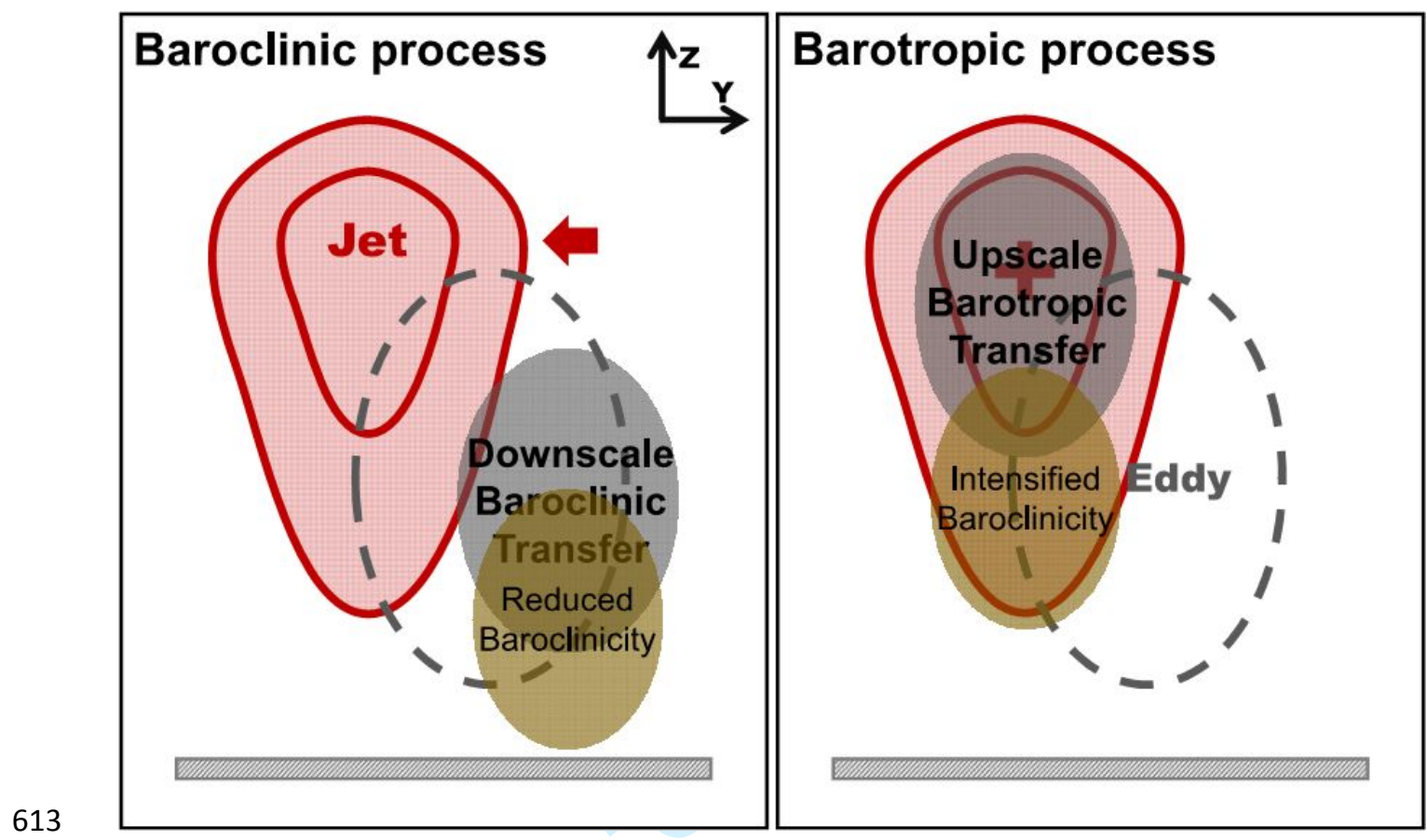

614 Figure 16. Schematic of the typical eddy feedback in the North Pacific storm track: (left)

615 the bacroclinic processes, and (right) the barotropic processes. Red and dashed gray

616 contours denote the jet and the synoptic eddy, respectively. The gray shaded area stands

617 for the canonical energy transfer centers, and the yellow shaded area are that with

618 baroclinicity change. The arrow in the left panel indicates the shrinking of the jet, and the

619 plus sign in the right panel indicates the jet acceleration.

620

621

622

623

624

\section{Conclusions}

Using a recently developed methodology, namely, the Multiscale Window Transform (MWT) and the MWT-based localized multiscale energetics analysis and vorticity analysis (MS-EVA), and an eddy tracking and compositing technique, this study investigates the general structure of the nonlinear interaction between the individual synoptic eddies and the jet stream over the North Pacific storm track, based on the 
626 ERA40 reanalysis data from ECWMF.

627 It is found that in the North Pacific storm track, more than 50\% (Type 1 and Type 4)

628 of the storms happen on the northern flank of the jet, about $40 \%$ are near the jet center,

629 with very few occurring south of the jet (less than 5\%). For the latter, the baroclinic

630 canonical transfer is rather weak. For the former two types of storms (near or to the north

631 of the jet center), their interaction with the background flow is quite asymmetric in space.

632 Specifically, the storms experience in the north a strong downscale canonical transfer of

633 APE, which weakens the baroclinicity and the background wind, while in the south, a

634 strong upscale canonical transfer of KE happens at the jet core which accelerates the jet

635 and enhances the baroclinicity. The resultant effect is that the jet strengthens but narrows,

636 resulting in an anomalous dipolar pattern in the background wind and baroclinicity field.

637 The general interaction scenario is schematized in Figure 16. (Note that the response of

638 the jet may also be partially due to other processes such as large scale energy fluxes.) On

639 average, the local interaction begins from about 3 days before a storm arrives at the site

640 of observation, achieves its maximum as the storm arrives, and then weakens.

641 In this study, the eddy-mean flow interaction has been investigated within the whole

642 north Pacific storm track throughout the entire cold season (October-April) without

643 considering the spatiotemporal variability, which is worth further investigation. As we

644 know, the strength and latitudinal location of the jet change remarkably with time

645 throughout the year (e.g., the jet is strong and located more equatorward in winter

646 whereas weak and located more poleward in summer). Changes in the jet's strength and 
647 latitudinal location may lead to different eddy-mean flow interactions (Chang, 2001a; 648 Harnik and Chang, 2004; Nakamura, 1992; Penny et al., 2013; Zhao and Liang, 2018).

649 Besides, the eddy-mean flow interaction in the Atlantic sector may have a different 650 scenario, which is for sure worth investigating. Previous studies have shown that the 651 Pacific jet is more subtropical (Lee and Kim, 2003) whereas the Atlantic jet is largely 652 eddy driven (Woollings et al., 2010). Both the Atlantic jet and storm track are more 653 southwest-northeast titled than their counterparts over the Pacific. Moreover, it has been 654 shown (Franzke et al., 2011; Novak et al., 2015; Woollings et al., 2010) that the 655 wintertime North Atlantic jet exhibits significant latitudinal variability, with evidence of 656 three preferred latitudinal locations: south, middle, and north; the corresponding 657 storm-track characteristics may be quite different, suggesting a probably more complex 658 eddy-mean flow interaction pattern in the Atlantic sector. We will explore these problems 659 in our next-step studies.

660

661 Appendix A. A brief introduction of multiscale window transform, canonical 662 transfer, and local Lorenz cycle

663 In this appendix, we present the needed mathematical formulas for the analysis. A 664 detailed introduction of the methodologies is beyond the scope here; for that purpose, 665 refer to Liang (2016) and Liang and Anderson (2007). In Xu and Liang (2017), there is a 666 brief comparison to the traditional energetics in the Reynolds framework.

667 A.1 Multiscale window transform 
668

669

670 decomposition-based formalisms as called, cannot be used to study the energy burst

671 processes which are generally localized in space and time. During past decades, a

672 common practice is to use filtering to replace the time averaging in these formalisms. For

673 example, application of a low-pass filter to a velocity field $u(t)$ yields a slowly varying $\bar{u}$

$674(t)$, and a high-pass filter to it gives a fast varying eddy part $u^{\prime}(t): u(t)=\bar{u}(t)+u^{\prime}(t)$.

675 Now time dependence is retained in both fields, and hence seemingly the local

676 information is retained. Entailing is, however, a very basic physical question: What are

677 the mean energy and eddy energy with this decomposition? When $\bar{u}$ is time invariant,

678 this is a Reynolds decomposition, we know the eddy energy is $\overline{\left[u^{\prime}(t)\right]^{2}}$; now if $\bar{u}$ is time

679 varying, what is the eddy energy then? During the past 2-3 decades, a common practice

680 in the literature is simply to take it as $\left[u^{\prime}(t)\right]^{2}$. That corresponds to, in the Reynolds

681 decomposition case, a relieving of the time mean in the eddy energy $\overline{\left[u^{\prime}(t)\right]^{2}}$.

682 This is, unfortunately, conceptually incorrect. To illustrate, consider a very simple

683 example which has a Fourier expansion

$$
u(t)=\bar{u}(t)+u^{\prime}(t)=\left[a_{0} \cos \omega_{0} t\right]+\left[a_{1} \cos \omega_{1} t\right], \omega_{1}>\omega_{0}
$$

684 with subscripts 0,1 representing the slow and fast processes, respectively. Now what are 685 the energies for these processes? In this simple case we know they should be, 686 respectively, $a_{0}^{2}$ and $a_{1}^{2}$. That is to say, multiscale energy is a concept in phase space; 687 here they are functions of the Fourier coefficients $\left(a_{0}, a_{1}\right)$. By the common practice with 
688

filters as shown above, however, the energies would be simply taken as

$$
[\bar{u}(t)]^{2}=\left[a_{0} \cos \omega_{0} t\right]^{2} \text { and }\left[u^{\prime}(t)\right]^{2}=\left[a_{1} \cos \omega_{1} t\right]^{2}
$$

689 which are functions of the reconstructions or filtered parts in physical space!

690 So the common practice during the past decades is conceptually incorrect. 691 Multiscale energy is a concept in phase space; it is related to its physical space 692 counterpart through the famous Parseval equality. Mathematically it is the square of the 693 norm of a field variable, or, alternatively, the Fourier transform of an autocorrelation 694 function (e.g., Batchelor, 1953). In the above example, when $\omega_{0}=0$, it is easy to prove 695 that $a_{1}{ }^{2}=\overline{\left[u^{\prime}(t)\right]^{2}}$, just as that with the Reynolds decomposition. From this example one 696 also sees that the time mean in the classical Reynolds formalism is essential; it cannot be 697 removed to pretend to gain the time variability. In fact, it is by no means a trivial task to 698 have the local energy of a time-dependent filtered field faithfully represented; it is a 699 rather profound problem. This even has not been possible until filter banks and wavelets 700 are connected (Strang and Nguyen, 1996), and has just been systematically addressed by 701 Liang and Anderson (2007) in the development of multiscale window transform (MWT). 702 703 orthogonal subspaces, each with an exclusive range of scales (represented by wavelet 704 scale levels), while having the local information retained. Such a subspace is termed a 705 scale window, or simply a window. MWT can be viewed as a generalization of the 706 classical Reynolds decomposition; originally it is developed for representing the energies 707 (and any quadratic quantities) on the resulting scale windows, in order to make multiscale 46 
708 energetics analysis possible. Liang and Anderson (2007) find that, for some specially 709 constructed orthogonal ${ }^{1}$ filters, there exists a transfer-reconstruction pair, namely, MWT 710 and its counterpart multiscale window reconstruction (MWR). In some sense MWR 711 functions just like a filter in the traditional sense. What makes it different is that, for each

712 MWR, there exists an MWT which gives coefficients that can be used to represent the 713 energy of the filtered series. In this way multiscale energetics analysis is made possible.

714 In MWT, a scale window is demarcated by two scale levels, or window bounds. For 715 a time series with a duration $\tau$, a scale level $j$ corresponds to a period $2^{-j} \tau$. In the $716 M$-window case, the windows are bounded above by $M+1$ scale levels: $j_{0}, j_{1}, \ldots, j_{M}$. 717 Alternatively, $2^{-j_{0}} \tau, 2^{-j_{1}} \tau, \ldots, 2^{-j_{M}} \tau$ are the time scale bounds. For convenience, we 718 will denote them by $\varpi=0,1, \ldots, M$ respectively.

719 Now suppose $\left\{\varphi_{n}^{j}(t)\right\}_{n}$ is an orthonormal translational invariant scaling sequence 720 (built from cubic splines; see Liang and Anderson (2007), and Figure 1 in Liang (2016)), 721 with $j$ some wavelet scale level and $n$ the time step. Let $T(t)$ be some square integrable 722 function defined on [0,1] (if not, the domain can always be rescaled to [0,1]). It has been 723 shown (Liang and Anderson 2007) that all such functions can be practically represented 724 using $\left\{\varphi_{n}^{j}(t)\right\}_{n}$ as a basis. In doing this, there is a scaling transform

$$
\hat{T}_{n}^{j}=\int_{0}^{1} T(t) \varphi_{n}^{j}(t) d t
$$

\footnotetext{
${ }^{1}$ Note here orthogonality is crucial; otherwise Parseval's relation does not hold and hence energy cannot even be defined.
} 
725 for any scale level $j$ (corresponding to frequency $2^{j}$ ). Given window bounds $j_{0}, j_{1}$ for a 726 two-window decomposition, $T$ then can be reconstructed on the windows formed above:

$$
\begin{aligned}
T^{\sim 0}(t) & =\sum_{n=0}^{2^{j_{0}}-1} \hat{T}_{n}^{j_{0}} \varphi_{n}^{j_{0}}(t), \\
T^{\sim 1}(t) & =\sum_{n=0}^{2^{j_{1}}-1} \hat{T}_{n}^{j_{1}} \varphi_{n}^{j_{1}}(t)-T^{\sim 0}(t),
\end{aligned}
$$

727 with the notations $\sim 0, \sim 1$ signifying the corresponding scale windows. With these

728 reconstructions (multiscale window reconstruction, or MWR for short), the MWT of $T$ is

729 defined as

$$
\hat{T}_{n}^{\sim \omega}=\int_{0}^{1} T^{\sim \omega}(t) \varphi_{n}^{j_{1}}(t) d t
$$

730 for windows $\varpi=0,1$ and $n=0,1, \cdots, N$, and $N=2^{j_{1}}-1$. In terms of $\hat{T}^{\sim \varpi}$ the above

731 reconstructions on the two windows can be written in a unified way:

$$
T^{\sim \varpi}(t)=\sum_{n=0}^{2^{j_{1}}-1} \hat{T}_{n}^{\sim \varpi} \varphi_{n}^{j_{1}}(t)
$$

732 These two equations make a transform-reconstruction pair for the MWT. Note that the $733 T^{\sim \varpi}(t)$ are just like the low/high-pass filtered quantities which are defined in physical 734 space, while the transform coefficients $\hat{T}_{n}^{\sim \varpi}$ (just like Fourier coefficients) can be used 735 to represent multiscale energy---it has been rigorously proved that the energy on scale $\varpi$ 736 is precisely equal to the square of the MWT coefficients (up to some constant multiplier).

737 Note it is by no means as trivial as $\left[T^{\sim \omega}(t)\right]^{2}$, which has been frequently seen in the 738 literature.

739 A.2 Multiscale energetics and local Lorenz cycle 
741 frame:

$$
\begin{aligned}
& \frac{\partial \mathbf{v}_{h}}{\partial t}+\mathbf{v}_{h} \cdot \nabla_{h} \mathbf{v}_{h}+\omega \frac{\partial \mathbf{v}_{h}}{\partial p}+f \mathbf{k} \times \mathbf{v}_{h}=-\nabla_{h} \Phi+\mathbf{F} \\
& \frac{\partial \Phi}{\partial p}=-\alpha \\
& \nabla_{h} \cdot \mathbf{v}_{h}+\frac{\partial \omega}{\partial p}=0 \\
& \quad \frac{\partial T}{\partial t}+\mathbf{v}_{h} \cdot \nabla_{h} \mathrm{~T}+\omega \frac{\partial T}{\partial p}+\omega \bar{\alpha} \frac{L-L_{d}}{g}+\omega \alpha \frac{L-L_{d}}{g}=\frac{\dot{q}_{n e t}}{c_{p}} \\
& p \alpha=R T
\end{aligned}
$$

742 where $L$ is the lapse rate and $L_{d}$ the lapse rate for dry air, the subscript $\mathrm{h}$ stands for 743 horizontal direction and the overbar for mean over time and over the isobaric plane. The 744 other notations are conventional. Note here $\Phi$ (geopotential) and $\alpha$ (specific volume) are 745 anomalies; their time averages have been pre-subtracted.

746 With MWT, the available potential energy (APE) and kinetic energy (KE) densities 747 (for convenience, we will simply refer to them as APE and KE, unless confusion may 748 arise) on window $\varpi$ at location $n$ can be defined, following Lorenz (1955), as

$$
\begin{aligned}
A^{\varpi} & =\frac{1}{2} c\left(\hat{T}^{\sim \varpi}\right)^{2}, \\
K^{\varpi} & =\frac{1}{2} \hat{\boldsymbol{v}}_{\mathrm{h}}^{\sim \varpi} \cdot \hat{\boldsymbol{v}}_{\mathrm{h}}^{\sim \varpi} .
\end{aligned}
$$

749 Note here the subscript $n$ has been suppressed for clarity; same below. From Eqs. 750 (A1)-(A5) the evolutionary equations for $K^{\varpi}$ and $A^{\varpi}$ for windows $\varpi(=0,1, \ldots)$ can 751 be obtained; they are Eqs. (1) and (2), which we rewrite as follows:

$$
\frac{\partial K^{\varpi}}{\partial t}+\nabla \cdot \mathbf{Q}_{K}^{\varpi}=\Gamma_{K}^{\varpi}-\nabla \cdot \mathbf{Q}_{P}^{\varpi}+b^{\varpi}+\mathrm{F}_{K}^{\varpi}
$$




$$
\frac{\partial A^{\varpi}}{\partial t}+\nabla \cdot \mathbf{Q}_{A}^{\varpi}=\Gamma_{A}^{\varpi}-b^{\varpi}+\mathrm{S}_{A}^{\varpi}+\mathrm{F}_{A}^{\varpi}
$$

752 The physical explanations and mathematical expressions for these terms are listed in

753 Table A1.

754

755 Table A1. The energetic terms in Equations (A8)-(A9). The colon operator (:) in $\Gamma_{K}^{\Phi}$ and

$756 \Gamma_{A}^{\varpi}$ is defined such that, for two dyadic products $\mathbf{A B}$ and $\mathbf{C D},(\mathbf{A B}):(\mathbf{C D})=(\mathbf{A} \cdot \mathbf{C})(\mathbf{B}$.

757 D). If total energetics (in W) are to be computed, the resulting integrals with respect to ( $x$,

$758 y, p$ ) should be divided by $g$. Besides, all terms are to be multiplied by $2^{j_{1}}$, which is

759 omitted for notational simplicity.

\begin{tabular}{|c|c|c|}
\hline Symbol & Mathematical expression & Physical interpretation \\
\hline$K^{\varpi}$ & $\frac{1}{2} \hat{\mathbf{v}}_{h}^{\sim \varpi} \cdot \hat{\mathbf{v}}_{h}^{\sim \varpi}$ & KE on scale window $\varpi$ \\
\hline $\boldsymbol{Q}_{K}^{\varpi}$ & $\frac{1}{2}\left(\widehat{\mathbf{v v}}_{\boldsymbol{h}}\right)^{\sim \varpi} \cdot \hat{\mathbf{v}}_{h}^{\sim \varpi}$ & Flux of KE on window $\varpi$ \\
\hline$\Gamma_{K}^{\varpi}$ & $\frac{1}{2}\left[\left(\widehat{\mathbf{v v}_{\boldsymbol{h}}}\right)^{\sim \varpi}: \nabla \hat{\mathbf{v}}_{h}^{\sim \varpi}-\nabla \cdot\left(\widehat{\mathbf{v v}_{\boldsymbol{h}}}\right)^{\sim \varpi} \cdot \hat{\mathbf{v}}_{h}^{\sim \varpi}\right]$ & $\begin{array}{l}\text { Canonical transfer of } \mathrm{KE} \text { to } \\
\text { window } \varpi\end{array}$ \\
\hline $\boldsymbol{Q}_{P}^{\varpi}$ & $\hat{\mathbf{v}}^{\sim \varpi} \hat{\Phi}^{\sim \varpi}$ & Geopotential flux \\
\hline$b^{\varpi}$ & $-\hat{\omega}^{\sim \varpi} \hat{\alpha}^{\sim \varpi}$ & Buoyancy conversion \\
\hline$A^{\varpi}$ & $\frac{1}{2} c\left(\hat{\mathrm{T}}^{\sim \varpi}\right)^{2}, c=\frac{g}{\bar{T}\left(g / C_{p}-L\right)}$ & APE on scale window $\varpi$ \\
\hline $\boldsymbol{Q}_{A}^{\varpi}$ & $\frac{1}{2} c \hat{\mathrm{T}}^{\sim \varpi}(\widehat{\mathbf{v T}})^{\sim \varpi}$ & Flux of APE on window $\varpi$ \\
\hline$\Gamma_{A}^{\Phi}$ & $\frac{c}{2}\left[(\widehat{\mathbf{v T}})^{\sim \varpi} \cdot \nabla \hat{\mathrm{T}}^{\sim \varpi}-\hat{\mathrm{T}}^{\sim \varpi} \nabla \cdot(\widehat{\mathbf{v T}})^{\sim \varpi}\right]$ & $\begin{array}{l}\text { Canonical transfer of APE to } \\
\text { window } \varpi\end{array}$ \\
\hline $\mathrm{S}_{A}^{\varpi}$ & $\frac{1}{2} \hat{\mathrm{T}}^{\sim \varpi}(\widehat{\omega \mathrm{T}}) \sim \varpi \frac{\partial c}{\partial p}+\frac{1}{\overline{\bar{T}}} \widehat{\omega \alpha}^{\sim \varpi}$ & $\begin{array}{l}\text { Apparent source/sink (usually } \\
\text { negligible) }\end{array}$ \\
\hline
\end{tabular}


761 Among those terms in Table $\mathrm{A} 1$ are $\Gamma_{\mathrm{A}}^{\varpi}$ and $\Gamma_{\mathrm{K}}^{\varpi}$, which represent the transfers of

762 APE and KE between the scale windows and hence make the processes that we are most

763 interested in for this study. For a scalar field $T$ (may be any scalar field or component of

764 vector field; not necessarily temperature) in a flow $v$, the energy transfer from other

765 scale windows to window $\varpi$ is (see Liang (2016) for a rigorous proof)

$$
\Gamma_{n}^{\varpi}=-E_{n}^{\varpi} \nabla \cdot \boldsymbol{v}_{\mathrm{T}}^{\varpi}=\frac{1}{2}\left[\widehat{(\boldsymbol{v} T)_{n}^{\sim \varpi}} \cdot \nabla \hat{T}_{n}^{\sim \varpi}-\hat{T}_{n}^{\sim \varpi} \nabla \cdot(\widehat{\boldsymbol{v} T})_{n}^{\sim \varpi}\right]
$$

766 where $E^{\varpi}=\frac{1}{2}\left(\hat{T}^{\sim \varpi}\right)^{2}$ is the energy of window $\varpi .{ }^{1}$ The other symbol

$$
\boldsymbol{v}_{\mathrm{T}}^{\varpi}=\frac{(\widehat{T \boldsymbol{v}})_{n}^{\sim \varpi}}{\hat{T}_{n}^{\sim \varpi}}
$$

767 is referred to as the T-coupled velocity, which can be understood as the weighted average

768 of $v$ in the phase space of MWT. With this, the transfer of APE and KE can be easily

769 obtained. For example,

$$
\Gamma_{\mathrm{K}}^{\varpi}=-\frac{1}{2}\left[\left(\hat{u}^{\sim \varpi}\right)^{2} \nabla \cdot \boldsymbol{v}_{\mathrm{u}}^{\varpi}+\left(\hat{v}^{\sim \varpi}\right)^{2} \nabla \cdot \boldsymbol{v}_{\mathrm{v}}^{\varpi}\right]
$$

770 which can be proved to be that in Table A1.

771 The so-obtained transfer possesses a very interesting property, namely

$$
\sum_{\varpi} \sum_{n} \Gamma_{n}^{\varpi}=0
$$

772 as first proposed in Liang and Robinson (2005) and later proved in Liang (2016).

773 Physically, this implies that the transfer is a mere redistribution of energy among the

774 scale windows, without generating or destroying energy as a whole. This property,

\footnotetext{
${ }^{1}$ When needed, a constant should be multiplied on both sides. For example, if $T$ is temperature, then $E^{\varpi}$ and $\Gamma^{\varpi}$ should be both multiplied by $c$ to make APE and APE transfer.
} 
775 though simple to state, does not hold in previous time decomposition-based or 776 Lorenz-type energetics formalisms (refer to Liang and Robinson (2007) for a clear 777 comparison to the classical formalism). To distinguish it from those that may have been 778 encountered in the literature, it is termed canonical transfer.

779 As shown in Liang (2016), a canonical transfer has a Lie bracket form; it satisfies 780 the Jacobian identity, reminiscent of the Poisson bracket in Hamiltonian mechanics. It 781 also satisfies a "detailed balance relation", which usually results from the Saltzman-type 782 or space decomposition-based energetics formalisms (Liang and Robinson 2005).

783 So, for an ideal fluid, the energetic processes represented in (A8)-(A9) are all 784 conservative. In other words, a local Lorenz cycle in the absence of dissipation and 785 diffusion is composed of the following three types of processes:

786 transports: redistributing energy in space (vanishing if integrated over a closed 787 domain),

- canonical transfers: redistributing energy among scale windows (vanishing if $789 \quad$ summarized over scale windows and locations),

790 buoyancy conversion: redistributing energy between APE and KE.

791 Figure A1 schematizes these processes for a two-window decomposition. Note here the 792 arrows connecting $K^{0}\left(A^{0}\right)$ and $K^{1}\left(A^{1}\right)$ are the quantities that are difficult to diagnose 793 with the traditional methods. 
794

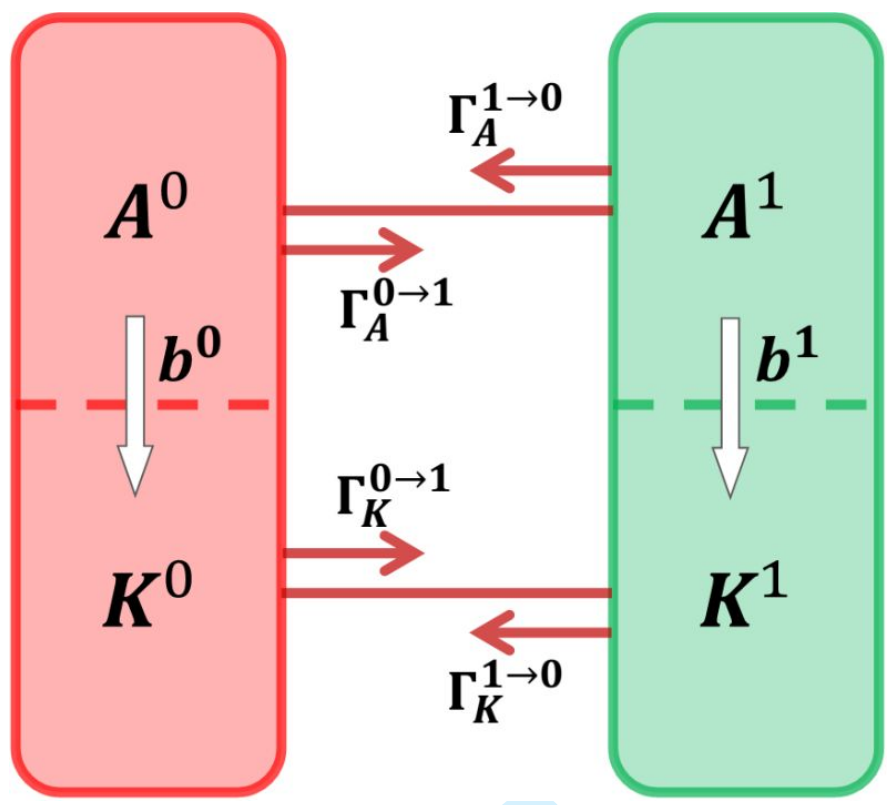

795 Figure A1. The local Lorenz cycle for a two-window decomposition (the scale windows 796 are denoted in the superscripts as 0 and 1, respectively). For clarity, transports and 797 dissipative/diffusive processes are not shown.

Note a canonical transfer may involve contributions from essentially all scale 799 windows; it is necessary to differentiate them. Consider for an example window $\varpi=1$. 800 The energy transferred to the window can be from window 0 and itself $\varpi=1$. Since all 801 canonical transfers are linear combinations of terms in a triple product form:

$$
\Gamma_{\mathrm{n}}^{1}=\hat{\mathcal{R}}_{n}^{\sim 1} \widehat{(p q)_{n}} \sim 1
$$

802 it suffices to consider $\Gamma_{\mathrm{n}}^{1}$. From Liang (2016), it is

$$
\Gamma_{\mathrm{n}}^{1}=\hat{\mathcal{R}}_{n}^{\sim 1}\left[\left(\widetilde{p^{\sim 0} q^{\sim 0}}\right)_{n}^{\sim 1}+\left(\widetilde{p^{\sim 0} q^{\sim 1}}\right)_{n}^{\sim 1}+\left(\widetilde{p^{\sim 1} q^{\sim 0}}\right)_{n}^{\sim 1}\right]+\hat{\mathcal{R}}_{n}^{\sim 1}\left(\widetilde{p^{\sim 1} q^{\sim 1}}\right)_{n}^{\sim 1}
$$

803 where the first part on the right hand side is the canonical energy transfer from window 0

804 to window 1 ; write it as $\Gamma^{0 \rightarrow 1}$. The second part, denoted by $\Gamma^{1 \rightarrow 1}$, is the canonical energy 805 transfer to itself, which is usually very small. $\Gamma^{0 \rightarrow 1}$ is of particular importance in that it 
806 is usually related to the instability in geophysical fluid dynamics; in particular, $\Gamma_{\mathrm{A}}^{0 \rightarrow 1}$ and

$807 \Gamma_{\mathrm{K}}^{0 \rightarrow 1}$ are usually related to baroclinic instability and barotropic instability of the mean

808 flow. For this reason, in the text sometimes $\Gamma_{\mathrm{A}}^{0 \rightarrow 1}$ and $\Gamma_{\mathrm{K}}^{0 \rightarrow 1}$ have been referred to as

809 baroclinic canonical transfer and barotropic canonical transfer, respectively.

810

811 Acknowledgements

812 The suggestions of two anonymous reviewers are sincerely appreciated. This study was

813 partially supported by the Jiangsu Provincial Government through the 2015 Jiangsu

814 Program for Innovation Research and Entrepreneurship Groups and the Jiangsu Chair

815 Professorship to XSL, the National Natural Science Foundation of China under Grants

816 No. 41276032 and 41705024, and the National Program on Global Change and Air-Sea

817 Interaction [GASI-IPOVAI-06].

818

819

820

821

822

823

824

825

826

827

828

829

830

831

832

\section{References}

Anderson, D., Hodges, K.I., Hoskins, B.J., 2003. Sensitivity of Feature-Based Analysis Methods of Storm Tracks to the Form of Background Field Removal. Mon. Weather Rev. 131, 565-573. https://doi.org/10.1175/1520-0493(2003)131<0565:SOFBAM>2.0.CO;2

Andrews, D.G., McIntyre, M.E., 1978. Generalized Eliassen-Palm and Charney-Drazin Theorems for Waves on Axismmetric Mean Flows in Compressible Atmospheres. J. Atmospheric Sci. 35, 175-185. https://doi.org/10.1175/1520-0469(1978)035<0175:GEPACD >2.0.CO;2

Barnes, E.A., Hartmann, D.L., 2011. Rossby Wave Scales, Propagation, and the Variability of Eddy-Driven Jets. J. Atmospheric Sci. 68, 2893-2908. https://doi.org/10.1175/JAS-D-11-039.1

Barnes, J.R., Young, R.E., 1992. Nonlinear Baroclinic Instability on the Sphere: Multiple Life Cycles with Surface Drag and Thermal Damping. J. Atmospheric Sci. 49, 

861-878. https://doi.org/10.1175/1520-0469(1992)049<0861:NBIOTS >2.0.CO;2 Bengtsson, L., Hodges, K.I., Esch, M., Keenlyside, N., Kornblueh, L., Luo, J.-J., Yamagata, T., 2007. How may tropical cyclones change in a warmer climate? Tellus A 59, 539-561. https://doi.org/10.1111/j.1600-0870.2007.00251.x Bengtsson, L., Hodges, K.I., Keenlyside, N., 2009. Will Extratropical Storms Intensify in a Warmer Climate? J. Clim. 22, 2276-2301. https://doi.org/10.1175/2008JCLI2678.1

Blackmon, M.L., 1976. A Climatological Spectral Study of the $500 \mathrm{mb}$ Geopotential Height of the Northern Hemisphere. J. Atmospheric Sci. 33, 1607-1623. https://doi.org/10.1175/1520-0469(1976)033<1607:ACSSOT>2.0.CO;2

Blackmon, M.L., Lee, Y.-H., Wallace, J.M., 1984. Horizontal Structure of $500 \mathrm{mb}$ Height Fluctuations with Long, Intermediate and Short Time Scales. J. Atmospheric Sci. 41 , 961-980 https://doi.org/10.1175/1520-0469(1984)041<0961:HSOMHF>2.0.CO;2

Blackmon, M.L., Wallace, J.M., Lau, N.-C., Mullen, S.L., 1977. An Observational Study of the Northern Hemisphere Wintertime Circulation. J. Atmospheric Sci. 34, $1040-1053$. https://doi.org/10.1175/1520-0469(1977)034<1040:AOSOTN >2.0.CO;2

Cai, M., M. Mak, 1990. On the Basic Dynamics of Regional Cyclogenesis. J. Atmospheric Sci., 47, 1417-1442, doi:10.1175/1520-0469(1990)047<1417:OTBDOR>2.0.CO;2.

Catto, J.L., Shaffrey, L.C., Hodges, K.I., 2010. Can Climate Models Capture the Structure of Extratropical Cyclones? J. Clim. 23, 1621-1635. https://doi.org/10.1175/2009JCLI3318.1

Chang, E.K.M., 2001a. GCM and Observational Diagnoses of the Seasonal and Interannual Variations of the Pacific Storm Track during the Cool Season. J. $\begin{array}{llrr}\text { Atmospheric } & \text { Sci. } & \text { 58, }\end{array}$ https://doi.org/10.1175/1520-0469(2001)058<1784:GAODOT >2.0.CO;2

Chang, E. K. M., 2001b. The Structure of Baroclinic Wave Packets. J. Atmospheric Sci., 58, 1694-1713, doi:10.1175/1520-0469(2001)058<1694:TSOBWP >2.0.CO;2.

Chang, E.K.M., 1993. Downstream Development of Baroclinic Waves As Inferred from Regression Analysis. J. Atmospheric Sci. 50, 2038-2053. https://doi.org/10.1175/1520-0469(1993)050<2038:DDOBWA>2.0.CO;2

Chang, E.K.M., Lee, S., Swanson, K.L., 2002. Storm Track Dynamics. J. Clim. 15, 2163-2183. https://doi.org/10.1175/1520-0442(2002)015<02163:STD>2.0.CO;2

Chang, E.K.M., Orlanski, I., 1993. On the Dynamics of a Storm Track. J. Atmospheric Sci. 50 , 999-1015. https://doi.org/10.1175/1520-0469(1993)050<0999:OTDOAS >2.0.CO;2

Charney, J.G., Drazin, P.G., 1961. Propagation of planetary-scale disturbances from the lower into the upper atmosphere. J. Geophys. Res. 66, 83-109. https://doi.org/10.1029/JZ066i001p00083 
874

875

876

877

878

879

880

881

882

883

884

885

886

887

888

889

890

891

892

893

894

895

896

897

898

899

900

901

902

903

904

905

906

907

908

909

910

911

912

913

914

Chen, G., Plumb, R.A., 2009. Quantifying the Eddy Feedback and the Persistence of the Zonal Index in an Idealized Atmospheric Model. J. Atmospheric Sci. 66, 37073720. https://doi.org/10.1175/2009JAS3165.1

Dacre, H.F., Hawcroft, M.K., Stringer, M.A., Hodges, K.I., 2012. An Extratropical Cyclone Atlas: A Tool for Illustrating Cyclone Structure and Evolution Characteristics. Bull. Am. Meteorol. Soc. 93, 1497-1502. https://doi.org/10.1175/BAMS-D-11-00164.1

Deng, Y., Mak, M., 2006. Nature of the Differences in the Intraseasonal Variability of the Pacific and Atlantic Storm Tracks: A Diagnostic Study. J. Atmospheric Sci. 63, 2602-2615. https://doi.org/10.1175/JAS3749.1

Dickinson, R.E., 1969. Theory of Planetary Wave-Zonal Flow Interaction. J. Atmospheric Sci. 26, 73-81. https://doi.org/10.1175/1520-0469(1969)026<0073:TOPWZF >2.0.CO;2

Edmon, H.J., Hoskins, B.J., McIntyre, M.E., 1980. Eliassen-Palm Cross Sections for the Troposphere. J. Atmospheric Sci. 37, 2600-2616. https://doi.org/10.1175/1520-0469(1980)037<2600:EPCSFT >2.0.CO;2

Eliassen, A., Palm, E., 1961. On the transfer of energy in stationary mountain waves. Geofys Publ 22, 1-23.

Farrell, B., 1984. Modal and Non-Modal Baroclinic Waves. J. Atmospheric Sci., 41, 668-673, doi:10.1175/1520-0469(1984)041<0668:MANMBW>2.0.CO;2.

_ 1985. Transient Growth of Damped Baroclinic Waves. J. Atmospheric Sci., 42, 2718-2727, doi:10.1175/1520-0469(1985)042<2718:TGODBW >2.0.CO;2.

_ , 1989. Optimal Excitation of Baroclinic Waves. J. Atmospheric Sci., 46, 1193 1206, doi:10.1175/1520-0469(1989)046<1193:OEOBW>2.0.CO;2.

Field, P.R., Wood, R., 2007. Precipitation and Cloud Structure in Midlatitude Cyclones. J. Clim. 20, 233-254. https://doi.org/10.1175/JCLI3998.1

Franzke, C., Woollings, T., Martius, O., 2011. Persistent Circulation Regimes and Preferred Regime Transitions in the North Atlantic. J. Atmospheric Sci. 68, 28092825. https://doi.org/10.1175/JAS-D-11-046.1

Gerber, E.P., Vallis, G.K., 2007. Eddy-Zonal Flow Interactions and the Persistence of the Zonal Index. J. Atmospheric Sci. 64, 3296-3311. https://doi.org/10.1175/JAS4006.1

Green, J.S.A., 1960. A problem in baroclinic stability. Q. J. R. Meteorol. Soc. 86, 237 251. https://doi.org/10.1002/qj.49708636813

Harnik, N., Chang, E.K.M., 2004. The Effects of Variations in Jet Width on the Growth of Baroclinic Waves: Implications for Midwinter Pacific Storm Track Variability. J. Atmospheric Sci. 23-40. https://doi.org/10.1175/1520-0469(2004)061<0023:TEOVIJ>2.0.CO;2

Hartmann, D.L., 1974. Time Spectral Analysis of Mid-Latitude Disturbances. Mon. Weather Rev. 102, 348-362. https://doi.org/10.1175/1520-0493(1974)102<0348:TSAOML $>2.0 . C O ; 2$ 
915

916

917

918

919

920

921

922

923

924

925

926

927

928

929

930

931

932

933

934

935

936

937

938

939

940

941

942

943

944

945

946

947

948

949

950

951

952

953

954

955

Hodges, K.I., 1995. Feature Tracking on the Unit Sphere. Mon. Weather Rev. 123, 34583465. https://doi.org/10.1175/1520-0493(1995)123<3458:FTOTUS $>2.0 . C O ; 2$

Hoskins, B.J., McIntyre, M.E., Robertson, A.W., 1985. On the use and significance of isentropic potential vorticity maps. Q. J. R. Meteorol. Soc. 111, 877-946. https://doi.org/10.1002/qj.49711147002

Hoskins, B.J., Valdes, P.J., 1990. On the Existence of Storm-Tracks. J. Atmospheric Sci. 47 ,

$1854-1864$. https://doi.org/10.1175/1520-0469(1990)047<1854:OTEOST>2.0.CO;2

James, I. N., 1987. Suppression of Baroclinic Instability in Horizontally Sheared Flows. J. Atmospheric Sci., 44, 3710-3720, doi:10.1175/1520-0469(1987)044<3710:SOBIIH>2.0.CO;2.

Kidston, J., Frierson, D.M.W., Renwick, J.A., Vallis, G.K., 2010. Observations, Simulations, and Dynamics of Jet Stream Variability and Annular Modes. J. Clim. 23, 6186-6199. https://doi.org/10.1175/2010JCLI3235.1

Kidston, J., Vallis, G.K., 2012. The Relationship between the Speed and the Latitude of an Eddy-Driven Jet in a Stirred Barotropic Model. J. Atmospheric Sci. 69, 32513263. https://doi.org/10.1175/JAS-D-11-0300.1

Lau, N.-C., 1979. The Structure and Energetics of Transient Disturbances in the Northern Hemisphere Wintertime Circulation. J. Atmospheric Sci. 36, 982-995. https://doi.org/10.1175/1520-0469(1979)036<0982:TSAEOT >2.0.CO;2

Lee, S., Kim, H., 2003. The Dynamical Relationship between Subtropical and Eddy-Driven Jets. J. Atmospheric Sci. 60, 1490-1503. https://doi.org/10.1175/1520-0469(2003)060<1490:TDRBSA >2.0.CO;2

Li, C., Wettstein, J.J., 2011. Thermally Driven and Eddy-Driven Jet Variability in Reanalysis. J. Clim. 25, 1587-1596. https://doi.org/10.1175/JCLI-D-11-00145.1

Liang, X.S., 2016. Canonical Transfer and Multiscale Energetics for Primitive and Quasigeostrophic Atmospheres. J. Atmospheric Sci. 73, 4439-4468. https://doi.org/10.1175/JAS-D-16-0131.1

Liang, X.S., Anderson, D.G.M., 2007. Multiscale Window Transform. Multiscale Model. Simul. 6, 437-467. https://doi.org/10.1137/06066895X

Liang, X.S., Robinson, A.R., 2007. Localized multi-scale energy and vorticity analysis. Dyn. Atmospheres Oceans 44, 51-76. https://doi.org/10.1016/j.dynatmoce.2007.04.001

Liang, X.S., Robinson, A.R., 2005. Localized multiscale energy and vorticity analysis. Dyn. Atmospheres Oceans 38, 195-230. https://doi.org/10.1016/j.dynatmoce.2004.12.004

Lim, G.H., Wallace, J.M., 1991. Structure and Evolution of Baroclinic Waves as Inferred from Regression Analysis. J. Atmospheric Sci. 48, 1718-1732. https://doi.org/10.1175/1520-0469(1991)048<1718:SAEOBW>2.0.CO;2

Lindzen, R.S., Farrell, B., 1980. A Simple Approximate Result for the Maximum Growth Rate of Baroclinic Instabilities. J. Atmospheric Sci. 37, 1648-1654. 
https://doi.org/10.1175/1520-0469(1980)037<1648:ASARFT>2.0.CO;2

Lindzen, R.S., Holton, J.R., 1968. A Theory of the Quasi-Biennial Oscillation. J. Atmospheric

Sci. 25, $1095-1107$. https://doi.org/10.1175/1520-0469(1968)025<1095:ATOTQB > 2.0.CO;2

Lorenz, D.J., 2014. Understanding Midlatitude Jet Variability and Change Using Rossby Wave Chromatography: Wave-Mean Flow Interaction. J. Atmospheric Sci. 71, 3684-3705. https://doi.org/10.1175/JAS-D-13-0201.1

Lorenz, D.J., Hartmann, D.L., 2001. Eddy-Zonal Flow Feedback in the Southern Hemisphere. J. Atmospheric Sci. 58, 3312-3327. https://doi.org/10.1175/1520-0469(2001)058<3312:EZFFIT>2.0.CO;2

Lorenz, E.N., 1955. Available Potential Energy and the Maintenance of the General Circulation. Tellus 7, 157-167. https://doi.org/10.1111/j.2153-3490.1955.tb01148.x

Ma, J., X.S. Liang, 2017. Multiscale dynamical processes underlying the wintertime Atlantic blockings. J. Atmos. Sci., 74, 3815-3831.

Mak, M., M. Cai, 1989. Local Barotropic Instability. J. Atmospheric Sci., 46, 3289-3311, doi:10.1175/1520-0469(1989)046<3289:LBI>2.0.CO;2.

Nakamura, H., 1992. Midwinter Suppression of Baroclinic Wave Activity in the Pacific. J. Atmospheric Sci. 49, 1629-1642. https://doi.org/10.1175/1520-0469(1992)049<1629:MSOBWA > 2.0.CO;2

Novak, L., Ambaum, M.H.P., Tailleux, R., 2015. The Life Cycle of the North Atlantic Storm Track. J. Atmospheric Sci. 72, 821-833. https://doi.org/10.1175/JAS-D-14-0082.1

Orlanski, I., Chang, E.K.M., 1993. Ageostrophic Geopotential Fluxes in Downstream and Upstream Development of Baroclinic Waves. J. Atmospheric Sci. 50, 212-225. https://doi.org/10.1175/1520-0469(1993)050<0212:AGFIDA>2.0.CO;2

Orlanski, I., Katzfey, J., 1991. The Life Cycle of a Cyclone Wave in the Southern Hemisphere. Part I: Eddy Energy Budget. J. Atmospheric Sci. 48, 1972-1998. https://doi.org/10.1175/1520-0469(1991)048<1972:TLCOAC $>2.0 . C O ; 2$

Papritz, L., S. Schemm, 2013. Development of an idealised downstream cyclone: Eulerian and Lagrangian perspective on the kinetic energy. Tellus Dyn. Meteorol. Oceanogr., 65, 19539, doi:10.3402/tellusa.v65i0.19539.

Penny, S.M., Battisti, D.S., Roe, G.H., 2013. Examining Mechanisms of Variability within the Pacific Storm Track: Upstream Seeding and Jet-Core Strength. J. Clim. 26, 5242-5259. https://doi.org/10.1175/JCLI-D-12-00017.1

Pierrehumbert, R.T., Swanson, K.L., 1995. Baroclinic Instability. Annu. Rev. Fluid Mech. 27, 419-467. https://doi.org/10.1146/annurev.f1.27.010195.002223

Plumb, R. A., 1983. A New Look at the Energy Cycle. J. Atmospheric Sci., 40, 16691688, doi:10.1175/1520-0469(1983)040<1669:ANLATE >2.0.CO;2.

Plumb, R.A., 1985. An Alternative Form of Andrews' Conservation Law for Quasi-geostrophic Waves on a Steady, Nonuniform Flow. J. Atmospheric Sci. 42, 
298-300. https://doi.org/10.1175/1520-0469(1985)042<0298:AAFOAC $>2.0 . C O ; 2$

Rivière, G., B. L. Hua, P. Klein, 2003. Perturbation growth in terms of barotropic alignment properties. Q. J. R. Meteorol. Soc., 129, 2613-2635, doi:10.1256/qj.02.106.

$\longrightarrow$, — and - 2004: Perturbation growth in terms of baroclinic alignment properties. Q. J. R. Meteorol. Soc., 130, 1655-1673, doi:10.1256/qj.02.223.

Rivière, G., P. Arbogast, A. Joly, 2015. Eddy kinetic energy redistribution within idealized extratropical cyclones using a two-layer quasi-geostrophic model: Eddy kinetic energy redistribution within idealized extratropical cyclones using a two-layer quasi-geostrophic model. Q. J. R. Meteorol. Soc., 141, 207-223, doi:10.1002/qj.2350.

Robinson, W.A., 2000. A Baroclinic Mechanism for the Eddy Feedback on the Zonal Index. J. Atmospheric Sci. 57, 415-422. https://doi.org/10.1175/1520-0469(2000)057<0415:ABMFTE $>2.0 . C O ; 2$

Simmons, A.J., Hoskins, B.J., 1980. Barotropic Influences on the Growth and Decay of Nonlinear Baroclinic Waves. J. Atmospheric Sci. 37, 1679-1684. https://doi.org/10.1175/1520-0469(1980)037<1679:BIOTGA>2.0.CO;2

Simmons, A.J., Hoskins, B.J., 1978. The Life Cycles of Some Nonlinear Baroclinic Waves. J. Atmospheric Sci. 35, 414-432. https://doi.org/10.1175/1520-0469(1978)035<0414:TLCOSN>2.0.CO;2

Sinclair, M.R., Revell, M.J., 2000. Classification and Composite Diagnosis of Extratropical Cyclogenesis Events in the Southwest Pacific. Mon. Weather Rev. 128 $1089-1105$. https://doi.org/10.1175/1520-0493(2000)128<1089:CACDOE >2.0.CO;2

Uppala, S.M., KÅllberg, P.W., Simmons, A.J., Andrae, U., Bechtold, V.D.C., Fiorino, M., Gibson, J.K., Haseler, J., Hernandez, A., Kelly, G.A., Li, X., Onogi, K., Saarinen, S., Sokka, N., Allan, R.P., Andersson, E., Arpe, K., Balmaseda, M.A., Beljaars, A.C.M., Berg, L.V.D., Bidlot, J., Bormann, N., Caires, S., Chevallier, F., Dethof, A., Dragosavac, M., Fisher, M., Fuentes, M., Hagemann, S., Hólm, E., Hoskins, B.J., Isaksen, L., Janssen, P. a. E.M., Jenne, R., Mcnally, A.P., Mahfouf, J.-F., Morcrette, J.-J., Rayner, N.A., Saunders, R.W., Simon, P., Sterl, A., Trenberth, K.E., Untch, A., Vasiljevic, D., Viterbo, P., Woollen, J., 2005. The ERA-40 re-analysis. Q. J. R. Meteorol. Soc. 131, 2961-3012. https://doi.org/10.1256/qj.04.176

Vallis, G.K., Gerber, E.P., 2008. Local and hemispheric dynamics of the North Atlantic Oscillation, annular patterns and the zonal index. Dyn. Atmospheres Oceans, Current Contributions to Understanding the General Circulation of the Atmosphere Part 2 44, 184-212. https://doi.org/10.1016/j.dynatmoce.2007.04.003

Woollings, T., Hannachi, A., Hoskins, B., 2010. Variability of the North Atlantic eddy-driven jet stream. Q. J. R. Meteorol. Soc. 136, 856-868. 
$1038 \quad$ https://doi.org/10.1002/qj.625

1039 Xu, F., Liang, X.S., 2017. On the generation and maintenance of the 2012/13 sudden 1040 stratospheric warming. J. Atmos. Sci., 74, 3209-3228.

1041 Zhang, Y., Yang, X.-Q., Nie, Y., Chen, G., 2012. Annular Mode-Like Variation in a 1042 Multilayer Quasigeostrophic Model. J. Atmospheric Sci. 69, 2940-2958. $1043 \quad$ https://doi.org/10.1175/JAS-D-11-0214.1

1044 Zhao, Y.-B., Liang X.S., 2018. On the inverse relationship between the boreal wintertime 1045 Pacific jet strength and storm-track intensity. J. Climate, $1046 \quad$ https://doi.org/10.1175/JCLI-D-18-0043.1, in press.

1047 